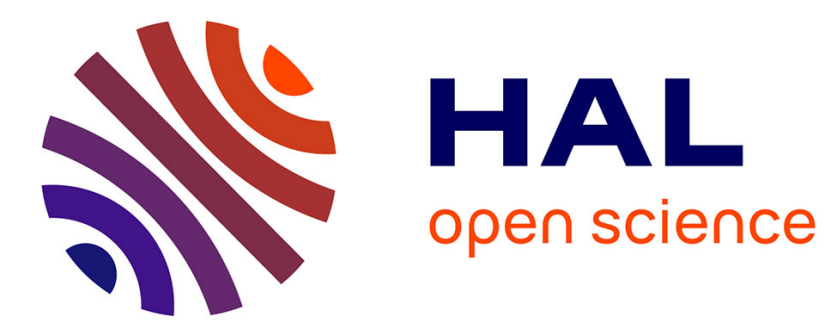

\title{
A synthetic forcing to trigger laminar-turbulent transition in parallel wall bounded flows via receptivity
}

Francesco Picella, Michele Alessandro Bucci, Stefania Cherubini, Jean-Christophe Robinet

\section{- To cite this version:}

Francesco Picella, Michele Alessandro Bucci, Stefania Cherubini, Jean-Christophe Robinet. A synthetic forcing to trigger laminar-turbulent transition in parallel wall bounded flows via receptivity. Journal of Computational Physics, 2019, 393, pp.92-116. 10.1016/j.jcp.2019.04.011 . hal-02462856

\section{HAL Id: hal-02462856 https://hal.science/hal-02462856}

Submitted on 31 Jan 2020

HAL is a multi-disciplinary open access archive for the deposit and dissemination of scientific research documents, whether they are published or not. The documents may come from teaching and research institutions in France or abroad, or from public or private research centers.
L'archive ouverte pluridisciplinaire HAL, est destinée au dépôt et à la diffusion de documents scientifiques de niveau recherche, publiés ou non, émanant des établissements d'enseignement et de recherche français ou étrangers, des laboratoires publics ou privés. 


\title{
Journal of Computational Physics
}

\section{A synthetic forcing to trigger laminar-turbulent transition in parallel wall bounded flows via receptivity}

\author{
Francesco Picella ${ }^{a}$, Michele Alessandro Bucci ${ }^{\mathrm{a}, \mathrm{b}}$, Stefania Cherubini ${ }^{\mathrm{c}}$, \\ Jean-Christophe Robinet ${ }^{\mathrm{a}, *}$ \\ a DynFluid Laboratory, Arts et Métiers, 151, Bd. de l'Hôpital, 75013, Paris, France \\ b Laboratoire d'informatique pour la mécanique et les sciences de l'ingénieur (LIMSI), Orsay, France \\ c DMMM, Politecnico di Bari, Via Re David 200, 70125 Bari, Italy
}

Keywords:

Laminar-turbulent transition

Wall bounded flows

Subcritical transition

\begin{abstract}
A B S T R A C T
Research on laminar-turbulent transition of wall-bounded parallel flows has usually focused on controlled scenarios where transition is triggered by perturbations having simple shapes and spectra. These disturbances strongly differ from the environmental noise usually present in experimental setups or industrial applications, where uncontrolled transition is usually observed. In this paper a new method is proposed to trigger uncontrolled transition to turbulence in wall-bounded parallel flows exploiting the receptivity of the flow to a volume forcing.

Using some concepts provided by linear stability and sensitivity analysis, such as the resolvent, we propose a method for constructing a volume forcing capable of inducing stochastic velocity perturbations with a prescribed energy level, eventually leading to laminar-turbulent transition as a response of the system to external noise.

The method has been tested in a channel flow configuration, using direct numerical simulations of the fully nonlinear Navier-Stokes equations in the presence of the volume forcing constructed on the basis of optimal forcing functions. Subcritical transition to turbulence induced by the prescribed forcing has been investigated and compared to other transition scenarios, where deterministic perturbations are imposed for obtaining a turbulent flow. Finally, the fully developed turbulent flows induced by the proposed method has been analysed, showing that low-order statistics and energy balance equations are practically unaffected by the continuous synthetic forcing.
\end{abstract}

\section{Introduction}

In this paper we present a new method to trigger turbulence in a streamwise-periodic shear flow using a synthetic forcing appropriately constructed on the basis of an optimal forcing analysis. Many techniques have been already developed to ensure a fully turbulent state when solving numerically the Navier-Stokes equations [1], such as the recycling inflow [2], the synthetic eddy [3] or the random Fourier method [4]. These approaches rely on ad-hoc inlet boundary conditions which are maintained during the course of the simulation, affecting the flow up to considerable distances downstream of it before reaching a fully turbulent regime. For this reason, these techniques are ill-suited for studying laminar-turbulent transition,

\footnotetext{
* Corresponding author.

E-mail address: jean-christophe.robinet@ensam.eu (J.-C. Robinet).
} 
since the footprints of these inlet conditions strongly affect the transition process. Differently from fully-developed turbulent flows which are characterized by time-decorrelated properties [5], the main features of transitional flows are strictly dependent on initial and boundary conditions, each imposed condition leading to a specific transition path which relies on different physical mechanisms [6]. For this reason, transition scenarios are classified depending on the nature of the initial and boundary conditions from which they stem: deterministic transition scenarios, that usually occur under controlled (laboratory) conditions, and stochastic ones, typical of uncontrolled configurations. The first class of transition paths is based on mechanisms that are well understood on a theoretical basis [7] including notably the K-type, H-type [8], Oblique Waves (OW) [9], and Streamwise Vortices (SV) [10] scenarios, to cite a few. Being mostly based on linear mechanisms at least in their early phases, they are quite easy to reproduce numerically although difficult to observe experimentally due to the low level of background noise requested [11]. On the other hand, when noise levels are sufficiently high, transition can occur bypassing some phases of linear growth of the disturbances, leading more rapidly to breakdown as a consequence of non-linear effects. This second class of transition paths, despite currently occurring in experiments in uncontrolled environments $[12,13]$, is challenging to reproduce numerically. For boundary-layer flows, the Free-Stream Turbulence (FST) typically occurring in experiments can be reproduced numerically by ad-hoc inlet boundary conditions using a numerical method introduced in Refs. [14,15]. This method, which has been specifically designed for wall-bounded open flows (namely, the flow over a flat plate), relies on the idea of injecting at the inlet of the numerical domain a velocity perturbation which accurately models the main features of free-stream turbulence, such as spectrum, turbulence intensity, and integral length scale. Due to the receptivity of the boundary layer, this disturbance will trigger transition downstream of the inlet, similarly to what happens in an experimental setup in the presence of grids. Numerically, this synthetic FST is constructed as a weighted sum of eigenmodes of the continuous branch of the Orr-Sommerfeld and Squire operator [15], with wavenumbers and associated amplitudes suitably chosen to satisfy a prescribed energy spectrum [14] in the homogeneous area outside the boundary layer. Using these methods, uncontrolled transition to turbulence in boundary-layer flows has been extensively studied [16-18] and compared to controlled scenarios [19,20,8,20,21], producing a rich body of literature [22,6]. On the other hand, in wall-bounded parallel flows such as the channel flow, although controlled transition has been long studied [23-25] (also due to the relatively low computational cost as compared to spatially evolving flows [26]), a little body of literature is available to the authors knowledge regarding uncontrolled transition. In fact, the presence of streamwise-periodic boundary conditions and the lack of a free-stream prevent the use of synthetic FST to induce uncontrolled transition in numerical experiments, which in most cases is triggered using noisy velocity fields built as a random superposition of Stokes modes [27]. However, despite being less studied, uncontrolled transition is far more common than controlled one, especially considering the fact that it usually occurs in subcritical conditions, namely for values of the Reynolds number lower than the critical linear one. Thus, new methods for numerically triggering this type of transition are worth to be introduced in the literature.

The aim of the present work is to design a method for triggering transition in a channel flow via response to a suitably constructed noisy perturbation mimicking those typically observed in uncontrolled transition. Due to the streamwise periodicity of the flow, this perturbation cannot be injected at the domain inlet, but it should be introduced in the NavierStokes equations as a forcing, in the same way as it is done for plasma actuators [28]. In many recent works [29,30], volume forcing is used to trigger turbulence in numerical simulations. Differently from these works, the method we propose is not specifically designed to efficiently obtain a turbulent state, but to induce uncontrolled transition in closed wall-bounded flows through receptivity of the flow to external disturbances, as it would occur in an experimental facility. In other words, the method proposed here intends to provide a numerical tool to trigger in a physically accurate way transition to turbulence, at the same time avoiding to impose a continuous forcing to sustain a constant turbulence level, as it usually occurs in unbounded flows [29].

In order to construct a perturbation able to trigger a large-amplitude response in the flow as a consequence of receptivity, we have chosen to use as a basis a set of harmonic disturbances that maximise (within a linear framework) the flow response for different given frequencies [31,32]. The usefulness of the optimal response to harmonic forcing, mediated by the resolvent operator, for constructing simple models reproducing key statistical and structural descriptions of wall turbulence, has been proven by many recent works (see [33,34] among others). The synthetic noisy volume forcing is then constructed as a linear composition of these optimal volume forcings issued from resolvent analysis, suitably weighted in order to respect a chosen energy spectrum.

In section 2 we set the problem framework and we outline the method for triggering turbulence in a streamwise-periodic wall-bounded flow by using a synthetic noisy volume forcing. In section 3 we test the method in a linearised framework to verify the consistency of all the assumptions previously made. In section 4 we provide a detailed description of the non-linear dynamics observed when transition is triggered using the method proposed here, with particular focus on the physical mechanisms as compared to classical transition scenarios. In section 5 we verify that the subsequent fully turbulent state is independent of the transition scenario from which it is generated. Finally, relevant conclusions are drawn in section 6.

\section{Problem statement and numerical methods}

The aim of this paper is to provide a new method for triggering uncontrolled transition in an incompressible wall-bounded streamwise-periodic flow such as a channel flow. This configuration has proven to be particularly suitable for numerical 
investigations of turbulent shear flows. In fact, being the problem homogeneous in the directions parallel to the wall, turbulent channel flows can be accurately simulated in relatively small domains [35] using periodic boundary conditions in both streamwise and spanwise directions [36]. The latter configuration, known as temporal channel flow [23], is chosen as framework of the present work due to its physical relevance, its large presence in the literature as well as to the low computational cost of numerical simulations in this setup as compared to spatially-evolving configurations such as the boundary-layer flow $[26,37]$.

Differently from several works already present in the literature, we are not interested in controlled transition scenarios, such as for instance the K-type one [24,25], but instead on investigating how transition arises as a response of the fluid system to external noise similar to that occurring in uncontrolled experimental conditions [38]. Thus, in the present work we will study how transition arises as a response of the flow to a synthetic noisy volume forcing. The latter, solution of the equations governing the fluid system, will be constructed using an ad hoc procedure relying on some concepts of linear stability and sensitivity analysis such as the resolvent analysis [31].

\subsection{Governing equations}

The dynamics of the incompressible flow of a Newtonian fluid are governed by the Navier-Stokes equations

$$
\begin{array}{r}
\frac{\partial \boldsymbol{U}}{\partial t}=-(\boldsymbol{U} \cdot \nabla) \boldsymbol{U}-\nabla P+\frac{1}{R e} \nabla^{2} \boldsymbol{U}+\boldsymbol{f} \\
\nabla \cdot \boldsymbol{U}=0,
\end{array}
$$

where $\boldsymbol{U}=(U(\boldsymbol{x}, t), V(\boldsymbol{x}, t), W(\boldsymbol{x}, t))^{T}$ is the velocity field, $P(\boldsymbol{x}, t)$ is the pressure and $\boldsymbol{f}$ the forcing field. The Reynolds number is defined as $R e=U H / v$, where $U$ is the centreline velocity, $H$ is half the height of the channel and $v$ the kinematic viscosity of the fluid. The reference frame $\boldsymbol{x}=(x, y, z)^{T}$ is chosen such that $x$ is the streamwise, $y$ the wall-normal and $z$ the spanwise directions.

Steady solutions $\boldsymbol{Q}_{b}(\boldsymbol{x})=\left(\boldsymbol{U}_{b}, P_{b}\right)^{T}$ of the Navier-Stokes equations are known as base flows or fixed points of the system. Under the assumption of small-amplitude disturbances, we decompose the flow field as a sum of the base flow and a perturbation such as $\boldsymbol{Q}(\boldsymbol{x}, t)=\boldsymbol{Q}_{b}(\boldsymbol{x})+\boldsymbol{q}(\boldsymbol{x}, t)$, that is to say $(\boldsymbol{U}(\boldsymbol{x}, t), P(\boldsymbol{x}, t))^{T}=\left(\boldsymbol{U}_{b}(\boldsymbol{x}), P_{b}(\boldsymbol{x})\right)^{T}+(\boldsymbol{u}(\boldsymbol{x}, t), p(\boldsymbol{x}, t))^{T}$. Linearising the governing equations around the base flow we obtain the linearized Navier-Stokes equations, which can be compactly written as:

$$
\frac{\partial \boldsymbol{u}}{\partial t}=\boldsymbol{L u}+\boldsymbol{f}
$$

once projected onto a divergence-free vector space. Being this system autonomous in time and being the base flow periodic in both streamwise and spanwise directions one can apply a Fourier transform to any field $\boldsymbol{q}$ so that $\boldsymbol{q}(\boldsymbol{x}, t)=\tilde{\boldsymbol{q}}(y) \exp [i(\alpha x+$ $\beta z)+\lambda t]+c . c$, where the last term stands for complex conjugate, $\alpha$ and $\beta$ are the streamwise and spanwise wavenumbers and $\lambda$ is the temporal one. In general $\alpha, \beta, \lambda, \tilde{\boldsymbol{u}} \in \mathbb{C}$.

When $\boldsymbol{f}=0$, the behaviour of a generic solenoidal velocity perturbation $\boldsymbol{u}$ is linked to the eigenpairs of $\boldsymbol{L}\left(\lambda^{\text {eig }}, \tilde{\boldsymbol{u}}^{\text {eig }}\right.$ ) [39], since it can be written as $\boldsymbol{u}(\boldsymbol{x}, t)=\sum_{l} \kappa_{l} \tilde{\boldsymbol{u}}(y)_{l}^{e i g} e^{i(\alpha x+\beta z)+\lambda_{l}^{e i g} t}$, where $\kappa_{l}$ is a scalar weight. For a given couple $\alpha, \beta \in \mathbb{R}$ (condition that will be held throughout the whole work), the temporal behaviour of each eigenvector of the linearised operator $\boldsymbol{L}$ is then described by its associated eigenvalue $\lambda_{i}^{e i g}=\sigma_{i}^{e i g}+i \omega_{i}^{e i g}$, where $\sigma_{i}^{e i g}$ represents its asymptotic growth/decay and $\omega_{i}^{e i g}$ its oscillation wavenumber.

We now assume the linearised system 3 to be forced harmonically in time as follows:

$$
\boldsymbol{f}(\boldsymbol{x}, t)=\tilde{\boldsymbol{f}}(y) e^{i \omega t} e^{i(\alpha x+\beta z)}+\text { c.c. }
$$

and that the system is stable, namely that all the eigenvalues of $\boldsymbol{L}$ have growth rate $\sigma_{i}^{\text {eig }}<0, \forall i$. Thanks to the linearity of the governing equations the Linear Time Invariant (LTI) system in equation (3) simplifies to:

$$
\tilde{\boldsymbol{u}}=(i \omega \boldsymbol{I}-\boldsymbol{L})^{-1} \tilde{\boldsymbol{f}},
$$

where $(i \omega \boldsymbol{I}-\boldsymbol{L})^{-1}$, called the resolvent of $\boldsymbol{L}$, represents a mapping between the harmonic input and the corresponding output for a given $[\alpha, \beta, \omega, R e]$ set and $\boldsymbol{I}$ stands for the identity operator. Under such forcing input the resulting response of the system will oscillate at the same frequency $\omega$, in particular:

$$
\boldsymbol{u}(\boldsymbol{x}, t)=\tilde{\boldsymbol{u}}(y) e^{i(\alpha x+\beta z)} e^{i \omega t},
$$

which is a monochromatic wave oscillating in time and space with wavenumbers $(\omega, \alpha, \beta)$. The kinetic energy density of this response for a given $[\alpha, \beta, \omega, \operatorname{Re}]$ set is defined as: 

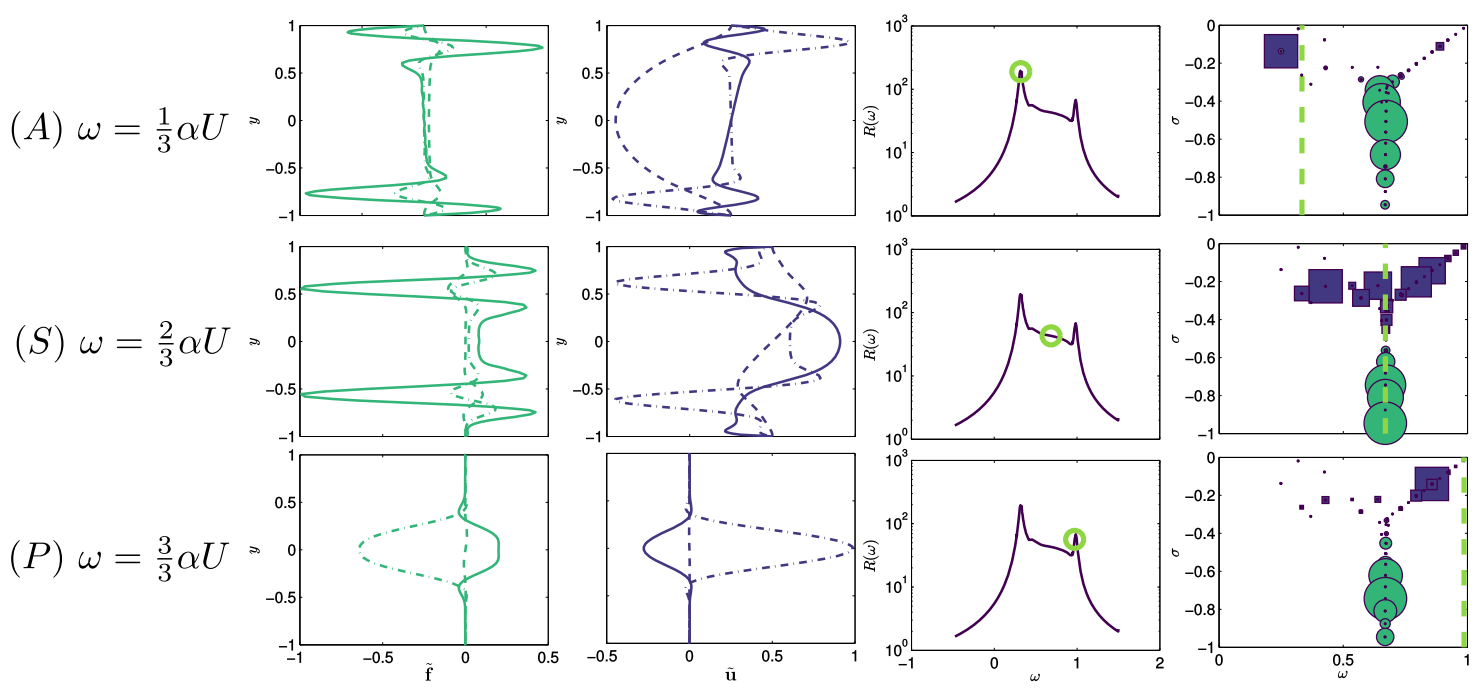

Fig. 1. Some typical optimal forcings/responses for Plane Poiseuille Flow (PPF) with $\alpha=1, \beta=0.25, R e=2000$, for various forcing frequencies $\omega: \omega=\alpha U / 3$ (upper frames), $\omega=2 \alpha U / 3$ (middle frames), and $\omega=\alpha U$ (bottom frames), triggering A-modes (viscous T-S modes), S-modes (critical layer modes) and P-modes (inviscid modes), respectively. From left to right the boxes represent the optimal forcing $\tilde{\boldsymbol{f}}^{\text {opt }}$, the optimal response $\tilde{\boldsymbol{u}}^{\text {opt }}$, the resolvent norm $R(\omega)$ highlighted as a circle, and the eigenspectrum $\lambda_{i}^{\text {eig }}$ of $\boldsymbol{L}$. The forcing and response vectors are built as a linear composition of the system's eigenvectors following equation (9), whose weights are proportional to the sizes of the markers. In the left frames solid, dashed, and dashed-dotted lines represent the streamwise, wall-normal, and spanwise velocity components, respectively.

$$
\|\tilde{\boldsymbol{u}}(y, \alpha, \beta, \omega)\|_{E}^{2}=\left.\frac{1}{2 V} \int_{V} \boldsymbol{u}^{H} \boldsymbol{u}(\boldsymbol{x}, t) d V\right|_{\alpha, \beta, \omega}=\left.E(\boldsymbol{u}(\boldsymbol{x}, t))\right|_{\alpha, \beta, \omega},
$$

where $V$ is the volume of the computational domain and $\boldsymbol{u}^{H}$ is the transconjugate of $\boldsymbol{u}$. Among all the possible $(\boldsymbol{f}, \boldsymbol{u})$ couples, let us focus on the one which maximizes the ratio of the output energy with respect to the input one, namely:

$$
R(\alpha, \beta, \omega)=\frac{\left\|\tilde{\boldsymbol{u}}^{o p t}\right\|_{E}^{2}}{\left\|\tilde{\boldsymbol{f}}^{o p t}\right\|_{E}^{2}}=\max _{\tilde{\boldsymbol{f}}} \frac{\|\tilde{\boldsymbol{u}}\|_{E}^{2}}{\|\tilde{\boldsymbol{f}}\|_{E}^{2}}=\left\|(i \omega \boldsymbol{I}-\boldsymbol{L})^{-1}\right\|_{E}^{2},
$$

where $\tilde{\boldsymbol{f}}^{\text {opt }}, \tilde{\boldsymbol{u}}^{\text {opt }}$ are defined as optimal forcing and response, again evaluated for a given $[\alpha, \beta, \omega, R e]$ set and $R$ is the resolvent norm. The optimal forcing and the associated response $\left(\tilde{\boldsymbol{f}}^{\text {opt }}, \tilde{\boldsymbol{u}}^{\text {opt }}\right)$ can be built using a linear combination of the eigenvectors of $\boldsymbol{L}$ :

$$
\begin{aligned}
\tilde{\boldsymbol{f}}^{\text {opt }}(y, \alpha, \beta, \omega, R e) & =\sum_{k=1}^{K} \bigcirc_{k} \tilde{\boldsymbol{f}}_{k}^{e i g}(y, \alpha, \beta, \omega, R e) \\
\tilde{\boldsymbol{u}}^{\text {opt }}(y, \alpha, \beta, \omega, \operatorname{Re}) & =\sum_{k=1}^{K} \square_{k} \tilde{\boldsymbol{u}}_{k}^{e i g}(y, \alpha, \beta, \omega, \operatorname{Re}),
\end{aligned}
$$

whose scalar weights $\left(\bigcirc_{k}, \square_{k}\right)$ are retrieved by means of a singular value decomposition of the resolvent norm [31,32]. An application of the resolvent norm theory to the case of plane Poiseuille flow is depicted in Fig. 1. In the reminder of this section we drop the Re dependency for the sake of readability.

\subsection{Building a volume forcing to enforce a prescribed energy spectrum}

Our aim is to construct a velocity perturbation attaining a target value of the turbulence intensity, $T u=\sqrt{\boldsymbol{U}_{r m s}^{2} / 3}$, which, within the linear framework, is equivalent to

$$
T u^{\text {linear }}=\sqrt{\boldsymbol{u}^{2} / 3} \equiv \sqrt{\frac{2}{3} E^{\text {linear }},}
$$

where $\boldsymbol{u}$ is the linear response to a given volume forcing.

Similarly to what is done in equation (9), we decompose the desired velocity perturbation field as a linear combination of a discrete set of optimal responses $\tilde{\boldsymbol{u}}^{\text {opt }}$, each one solution of equations (1) and (8). The associated optimal forcing fields are 
then used as a basis to construct the synthetic volume forcing able to induce the desired perturbation field via receptivity mechanisms. Optimal forcings are here chosen as a basis not only because they provide a straightforward force-velocity relation, but also because they are able to induce the given energy level $T u^{\text {linear }}$ with the lowest possible forcing intensity (within a linearized framework). The optimal forcing and response fields are computed using a simple 1D code solving equation (8) for different values of the wavenumbers $[\alpha, \beta, \omega]$, conveniently chosen to discretize the prescribed energy spectrum.

For the sake of simplicity, in the following we will assume a flat energy spectrum, whose energy is evenly distributed within a frequency range $\left[\omega_{\min }, \omega_{\max }\right]$, although the following procedure can be easily extended to more complex energy spectra (see section 3.3). The prescribed continuous energy spectrum is discretized into $N_{\omega}$ modes $\check{\boldsymbol{u}}_{i}$, each one associated to a specific frequency $\omega_{i}$, so that:

$$
\omega_{i}=\frac{\left(\omega_{\max }-\omega_{\min }\right)(i-1)}{N_{\omega}}+\omega_{\min }
$$

and

$$
\boldsymbol{u}(\boldsymbol{x}, t)=\sum_{i=1}^{N_{\omega}} \check{\boldsymbol{u}}\left(\boldsymbol{x}, \omega_{i}\right) e^{i \omega_{i} t}, \quad E\left(\check{\boldsymbol{u}}\left(\boldsymbol{x}, \omega_{i}\right)\right)=\frac{E^{\text {linear }}}{N_{\omega}}
$$

where the left side of equation (13) coincides with the Discrete Fourier Transform (DFT) of $\boldsymbol{u}(\boldsymbol{x}, t), \check{\boldsymbol{u}}\left(\boldsymbol{x}, \omega_{i}\right)$ being a discrete subset of DFT modes associated to its respective discrete set of forcing frequencies:

$$
\omega=\left[\omega_{\min }, \ldots, \omega_{i}, \ldots, \omega_{\max }\right] .
$$

We further decompose the velocity field associated to a given frequency $\check{\boldsymbol{u}}\left(\boldsymbol{x}, \omega_{i}\right)$ into $N_{\alpha, \beta}$ discrete modes for each allowed $(\alpha, \beta)$ spatial wavenumber. The physical reasons of this restriction will be explained in detail in section (2.3). Thus, the resolvent norm analysis will provide a discrete set of modes associated to $N_{\alpha, \beta}$ spatial wavenumbers:

$$
\alpha, \beta\left(\omega_{i}\right)=\left[(\alpha, \beta)_{i}^{1}, \ldots,(\alpha, \beta)_{i}^{j}, \ldots,(\alpha, \beta)_{i}^{N_{\alpha, \beta}}\right],
$$

each one compatible with a corresponding single frequency $\omega_{i}$. Similarly to equation (13), we can further decompose the velocity field as follows:

$$
\check{\boldsymbol{u}}\left(\boldsymbol{x}, \omega_{i}\right)=\sum_{j=1}^{N_{\alpha, \beta}} \tilde{\boldsymbol{u}}\left(y, \alpha_{i}^{j}, \beta_{i}^{j}, \omega_{i}\right) e^{i\left(\alpha_{i}^{j} x+\beta_{i}^{j} z\right)}, \quad\left\|\tilde{\boldsymbol{u}}\left(y, \alpha_{i}^{j}, \beta_{i}^{j}, \omega_{i}\right)\right\|_{E}^{2}=\frac{E^{\text {linear }}}{N_{\omega} N_{\alpha, \beta}}
$$

where each $\tilde{\boldsymbol{u}}\left(y, \alpha_{i}^{j}, \beta_{i}^{j}, \omega_{i}\right)$ has the same kinetic energy for the simple test case considered here. The couples $\left(\alpha_{i}^{j}, \beta_{i}^{j}\right)$ are suitably chosen to span a large number of wavelengths in order to increase the isotropy of the perturbation, similarly to FST methods $[15,14]$.

Replacing the generic $\tilde{\boldsymbol{u}}$ with $\tilde{\boldsymbol{u}}^{\text {opt }}$ in equation (16) results in:

$$
\check{\boldsymbol{u}}\left(\boldsymbol{x}, \omega_{i}\right)=\sum_{j=1}^{N_{\alpha, \beta}} \tilde{\boldsymbol{u}}^{o p t}\left(y, \alpha_{i}^{j}, \beta_{i}^{j}, \omega_{i}\right) e^{i\left(\alpha_{i}^{j} x+\beta_{i}^{j} z\right)}, \quad\left\|\tilde{\boldsymbol{u}}^{o p t}\left(y, \alpha_{i}^{j}, \beta_{i}^{j}, \omega_{i}\right)\right\|_{E}^{2}=\frac{E^{\text {linear }}}{N_{\omega} N_{\alpha, \beta}} .
$$

The same procedure is then applied to the discrete set of forcing fields associated to the chosen optimal responses, obtaining:

$$
\check{\boldsymbol{f}}\left(\boldsymbol{x}, \omega_{i}\right)=\sum_{j=1}^{N_{\alpha, \beta}} A_{i}^{j} \tilde{\boldsymbol{f}}^{o p t}\left(y, \alpha_{i}^{j}, \beta_{i}^{j}, \omega_{i}\right) e^{i\left(\alpha_{i}^{j} x+\beta_{i}^{j} z\right)} e^{i\left(\phi_{x} x+\phi_{z} z\right)},
$$

where each optimal forcing $\tilde{\boldsymbol{f}}^{\text {opt }}$ is multiplied by the scalar $A_{i}^{j}$ to ensure a prescribed weight and shifted in space by a random phase $\phi_{x}, \phi_{z}$ to increase homogeneity [14]. The weights $A_{i}^{j}$ are assigned in order to fulfil the prescribed energy spectrum; in particular, combining equations (8) and (17) one obtains:

$$
\frac{E^{\text {linear }}}{N_{\omega} N_{\alpha, \beta}}=A_{i}^{j}\left\|\tilde{\boldsymbol{u}}^{\text {opt }}\left(y, \alpha_{i}^{j}, \beta_{i}^{j}, \omega_{i}\right)\right\|_{E}^{2}=A_{i}^{j} R\left(y, \alpha_{i}^{j}, \beta_{i}^{j}, \omega_{i}\right)\left\|\tilde{\boldsymbol{f}}^{o p t}\left(y, \alpha_{i}^{j}, \beta_{i}^{j}, \omega_{i}\right)\right\|_{E}^{2},
$$

which is then rearranged to provide the value of $A_{i}^{j}$ for each forcing $\tilde{\boldsymbol{f}}^{\text {opt }}$ :

$$
A_{i}^{j}=\frac{E^{\text {linear }}}{N_{\omega} N_{\alpha, \beta} R\left(\alpha_{i}^{j}, \beta_{i}^{j}, \omega_{i}\right)} .
$$




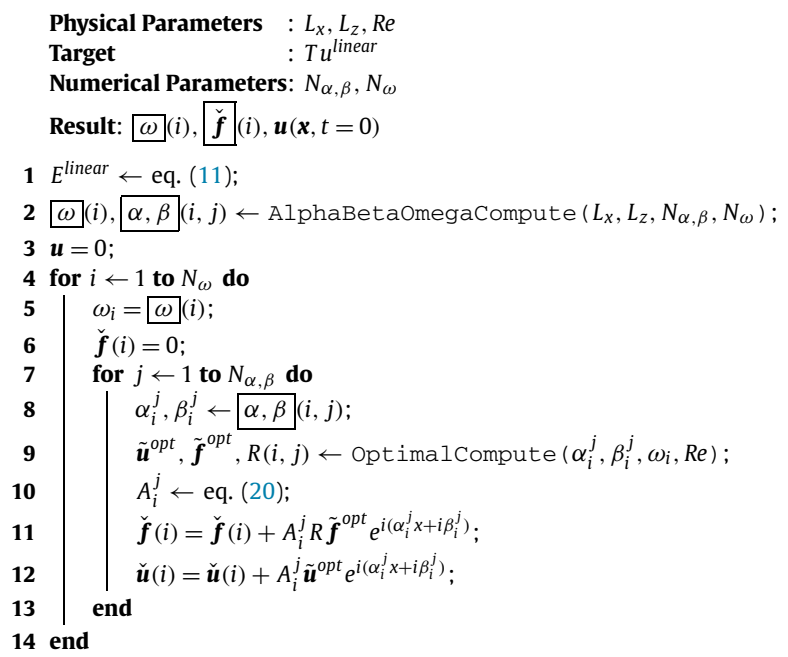

Algorithm 1: F-TYPE forcing method. The AlphaBetaOmegaCompute algorithm, detailed in algorithm (2) in section 2.3, provides the discrete $\omega_{i}$ and $\alpha_{i}^{j}, \beta_{i}^{j}$ sets for a given configuration, while the Optimalcompute function computes the 1D optimal forcing fields solving equation (8), as shown in [31]. The whole method is sketched in Fig. 2.

The obtained discrete set of $N_{\omega}$ forcing fields reads:

$$
\check{\boldsymbol{f}}\left(\omega_{i}\right)=\left[\check{\boldsymbol{f}}\left(\boldsymbol{x}, \omega_{\min }\right), \ldots, \check{\boldsymbol{f}}\left(\boldsymbol{x}, \omega_{i}\right), \ldots, \check{\boldsymbol{f}}\left(\boldsymbol{x}, \omega_{\max }\right)\right],
$$

where each forcing is associated to a given frequency $\omega_{i}$. The real volume forcing to be injected in equations (1) in order to obtain the requested response by time marching can be expressed as:

$$
\boldsymbol{f}(\boldsymbol{x}, t)=\sum_{i=1}^{N_{\omega}} \check{\boldsymbol{f}}\left(\boldsymbol{x}, \omega_{i}\right) e^{i \omega_{i} t}=\sum_{i=1}^{N_{\omega}}\left[\mathfrak{R}\left(\check{\boldsymbol{f}}\left(\boldsymbol{x}, \omega_{i}\right)\right) \cos \left(\omega_{i} t\right)-\check{\mathfrak{s}}\left(\check{\boldsymbol{f}}\left(\boldsymbol{x}, \omega_{i}\right)\right) \sin \left(\omega_{i} t\right)\right]
$$

Within a linearized framework, the obtained response reaching the prescribed energy level $T u^{\text {linear }}$ has the form:

$$
\boldsymbol{u}(\boldsymbol{x}, t)=\sum_{i=1}^{N_{\omega}} \check{\boldsymbol{u}}\left(\boldsymbol{x}, \omega_{i}\right) e^{i \omega_{i} t}=\sum_{i=1}^{N_{\omega}}\left[\Re\left(\check{\boldsymbol{u}}\left(\boldsymbol{x}, \omega_{i}\right)\right) \cos \left(\omega_{i} t\right)-\Im\left(\check{\boldsymbol{u}}\left(\boldsymbol{x}, \omega_{i}\right)\right) \sin \left(\omega_{i} t\right)\right] .
$$

The whole procedure, to which we will refer to as F-TYPE forcing method, is summarized in Algorithm 1 and in Fig. 2.

From a practical point of view, we first compute the set of $N_{\omega}$ forcing fields $\breve{f}(\boldsymbol{x}, t)$ in the frequency domain as a preprocessing, prescribing only the discrete set of $N_{\alpha, \beta}$ and $N_{\omega}$ wavenumbers. Then, we compute $\boldsymbol{f}$ at each timestep by means of equation (22) and feed it to the direct numerical simulation. It is noteworthy that, while the whole energy is equally distributed on the optimal response fields composing the desired velocity perturbation as imposed in equation (13), each optimal forcing can be associated to a different energy level, since the resolvent norm acting as input-output transfer function has a different value for each wavenumber/frequency. As a consequence, the energy spectrum of the forcing field will not be flat in $\omega$.

\subsection{Constraints on $\alpha, \beta$, and $\omega$}

As mentioned in the previous subsection, the optimal forcing fields used to construct the desired synthetic volume forcing for triggering uncontrolled transition, are function of $\alpha, \beta, \omega$. These parameters cannot be assigned freely, being linked to each other due to some physical reasons detailed below. The first reason is based on simple geometrical considerations: being the numerical setup periodic in both streamwise and spanwise directions, only a finite number of wavelengths are allowed within the assigned domain. Beyond the trivial 0th mode, the lowest possible wavelengths are therefore determined by the size of the computational domain $L_{x}, L_{z}$ through the relations $\alpha_{\text {box }}=2 \pi / L_{x}, \beta_{\text {box }}=2 \pi / L_{z}$. Integer multiples of these wavenumbers can be also retrieved, up to the maximum wavenumber allowed due to spatial discretization. Using the Nyquist-Shannon sampling theorem [40], one obtains $\alpha_{\max } \approx 2 \pi /(8 \Delta x),\left(\beta_{\max } \approx 2 \pi /(8 \Delta z)\right.$, providing the following discrete set of geometrically compatible wavelengths:

$$
\alpha{ }_{\text {geom }}=\left[0, \alpha_{\text {box }}, 2 \alpha_{\text {box }}, \ldots, \alpha_{\text {max }}\right], \quad \beta \beta_{\text {geom }}=\left[0, \beta_{\text {box }}, 2 \beta_{\text {box }}, \ldots, \beta_{\text {max }}\right]
$$




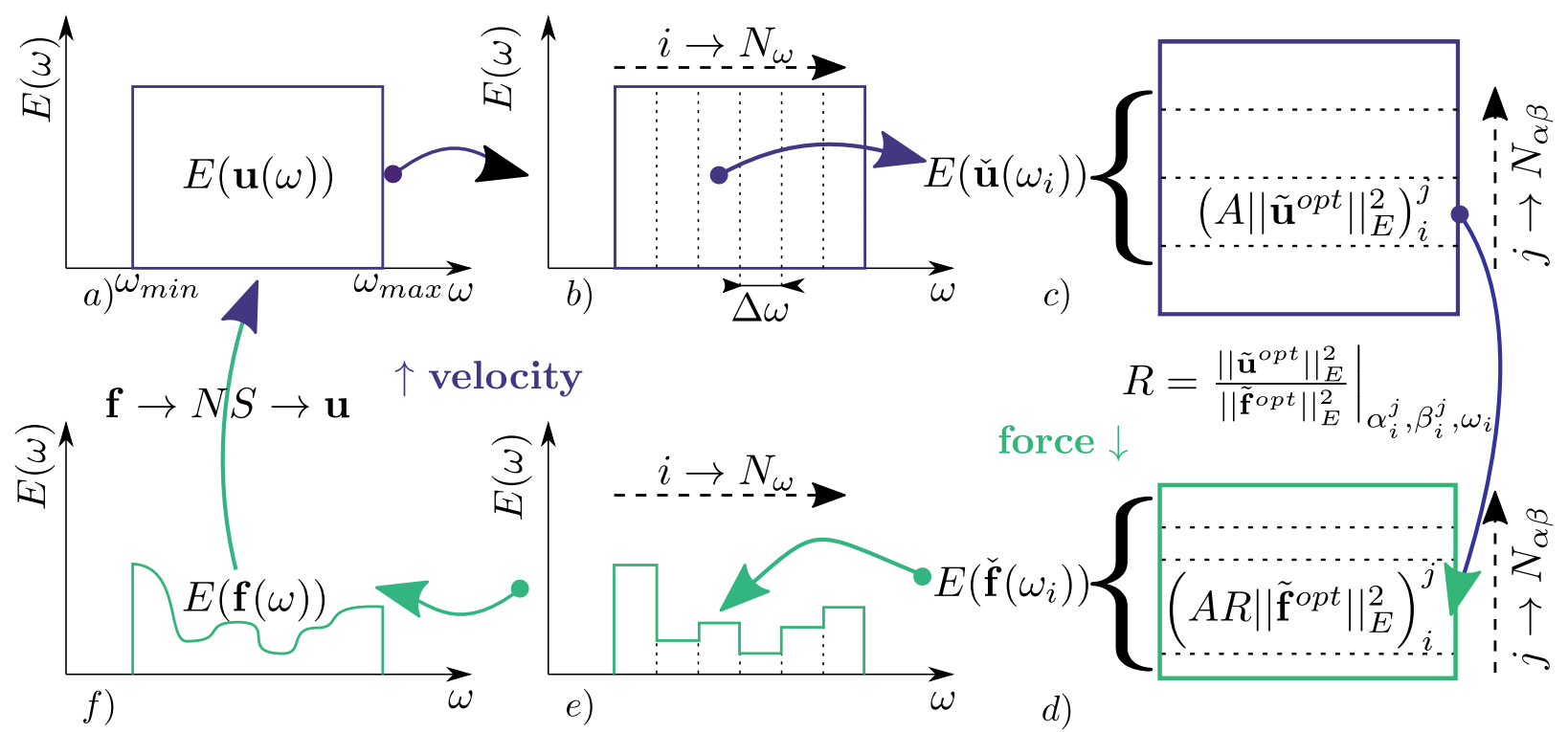

Fig. 2. Sketch depicting the F-type forcing method detailed in Algorithm 1, aiming to build a forcing which ensures a velocity perturbation field reaching $T u^{\text {linear. }}$.

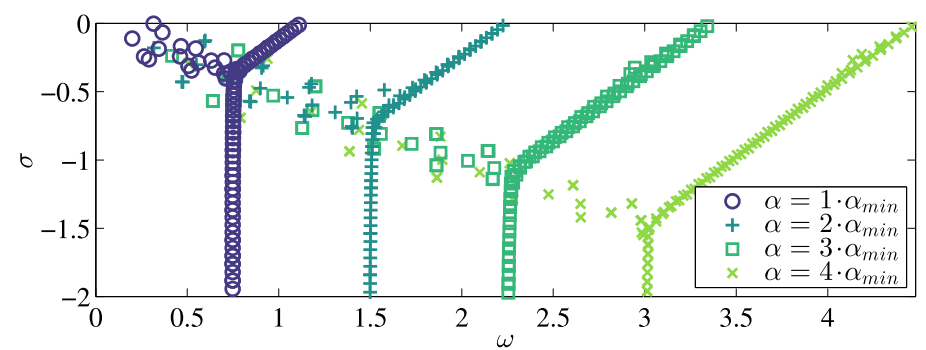

Fig. 3. Orr-Sommerfeld spectra of plane Poiseuille flow for $R e=5000, \beta=0$ and different values of $\alpha$ provided in the legend. The spectra widen as the streamwise wavenumber is increased. This physically means that shorter streamwise wavelengths can be linked to higher forcing frequencies. For this computation we have used a pseudo-spectral Chebyshev collocation method with numerical resolution of 256 points in the $y$ direction.

where $\Delta x, \Delta z$ indicate the characteristic grid size in the streamwise and spanwise directions, respectively. Concerning the wavenumber $\alpha=0$, although being geometrically allowed within the domain, it would result in a deformation of the one dimensional base flow, changing the physical nature of the problem. Thus, the allowed wavenumber set is reduced to:

$$
\begin{array}{r}
\alpha=\left[\alpha_{b o x}, 2 \cdot \alpha_{b o x}, \ldots, \alpha_{\max }\right] \\
\beta=\left[0, \beta_{b o x}, 2 \cdot \beta_{b o x}, \ldots, \beta_{\max }\right]
\end{array}
$$

The same can be said about the $\omega=0$ temporal mode, which would result in an unphysical constant forcing. The forcing frequencies will thus lie in the range $\left[\omega_{\min }, \omega_{\max }\right]$, where the values of $\omega_{\min }, \omega_{\max }$ can be easily chosen due to the following physical considerations. In Fig. 1 one can notice how the wall normal distribution of the modes changes depending on the assigned value of $\omega$ : different forcing frequencies result in different sensitivity mechanisms, which can be retrieved in the spectrum in the form of viscous Tollmien-Schlichting, critical layer, and inviscid modes (from upper to bottom frames). Similarly to FST method in semi-bounded domains [14,15] where only free-stream modes are excited, in the current setup only inviscid modes will be used, whereas the viscous Tollmien-Schlichting waves, also referred to as A-modes [31], are discarded. Towards this aim, the forcing frequencies associated to a particular spatial wavenumber are constrained within the range:

$$
\operatorname{range}(\omega(\alpha))=\left\{\frac{2}{3} \alpha, \alpha\right\} U
$$

where a generalisation of Taylor's hypothesis $\omega=\alpha U$ is used, $2 U / 3$ corresponding to the mean velocity of the flow. In this way, for a given $\alpha_{i}$, all the viscous A-modes are discarded, leaving only inviscid (P and S) modes to construct the desired volume forcing. Moreover, as depicted in Fig. 3, one can observe that a given $\omega$ range is associated to each $\alpha$. Thus, the 


\section{Table 1}

Lower and upper bounds for the spatial (left) and temporal (right) wavenumbers of the optimal forcing fields used for constructing the desired volume forcing.

\begin{tabular}{llllll}
\hline$\alpha_{\min }$ & $\alpha_{\max }$ & $\beta_{\min }$ & $\beta_{\max }$ & $\omega_{\min }$ & $\omega_{\max }$ \\
\hline$\frac{2 \pi}{L_{x}}$ & $\frac{2 \pi}{8 \Delta x}$ & 0 & $\frac{2 \pi}{8 \Delta x}$ & $\frac{2}{3} \alpha_{\min }$ & $\alpha_{\max }$ \\
\hline
\end{tabular}

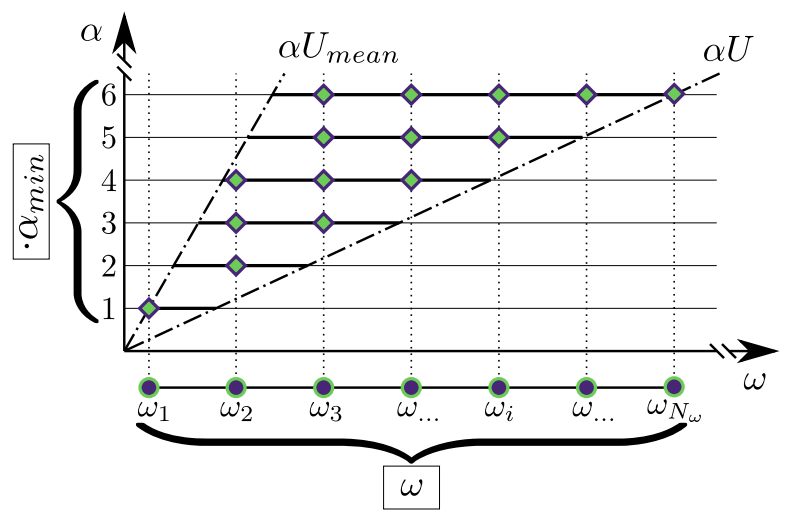

Fig. 4. Graphical summary of section 2.3. Diamonds and circles represent the spatial and temporal wavenumbers taken into account for building up the synthetic volume forcing.

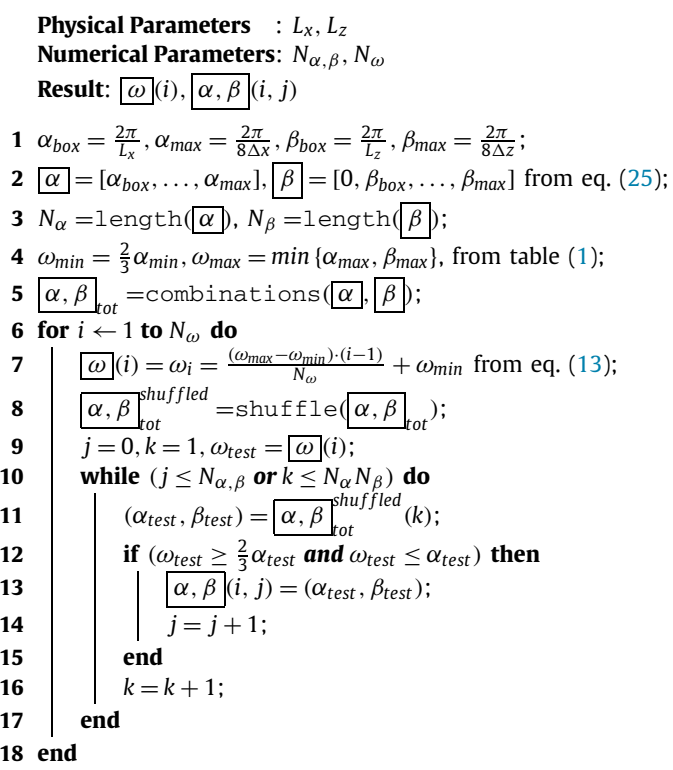

Algorithm 2: AlphaBetaOmegaCompute algorithm, which summarises the constraints on $\alpha, \beta$ for each discrete forcing frequency $\omega$ as detailed in section 2.3. Function length() computes the number of elements of an input discrete list $x$; function combinations() provides all their possible combinations; and function shuffle() randomly redistributes the elements of the list.

$\omega$ range results from $\alpha$, as reported in equation (27). Table 1 summarises the lower and upper bounds of the spatial and temporal wavenumbers of the optimal forcing fields used for constructing the desired volume forcing. It is noteworthy to remark that, while $\alpha$ depends on the specific forcing frequency $\omega_{i}, \beta$ is only dependent on the domain discretization.

This feature can result in a discontinuous spectrum for low forcing frequencies, depending on the domain sizes $L_{x}, L_{z}$.

A sketch of the allowed spatial and temporal wavenumbers is provided in Fig. 4, and the numerical procedure to choose them is summarised in Algorithm 2. 


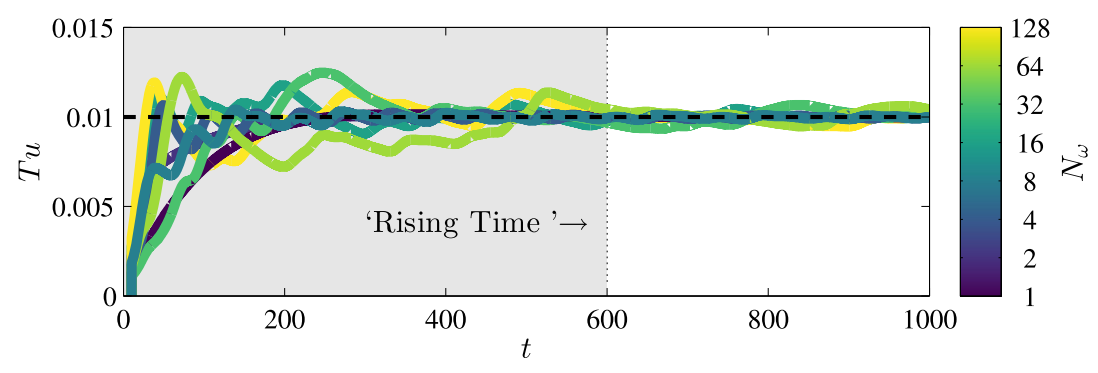

Fig. 5. Time evolution of turbulent intensity extracted from a linearised DNS forced by the F-type method with $T u^{\text {linear }}=0.01, \boldsymbol{u}(\boldsymbol{x}, t=0)=\mathbf{0}$, and for different $N_{\omega}$ and $N_{\alpha, \beta}=4$ as indicated in the legend. The prescribed turbulence intensity $T u^{\text {linear }}$ is attained after a finite rising time, when the transient behaviour has left place to an asymptotic regime. (For interpretation of the colours in the figure(s), the reader is referred to the web version of this article.)

\section{Algorithm testing: linear regime}

In the following we test and tune our method by running forced direct numerical simulations of equations (3). The Navier-Stokes equations are solved using the incompressible flow solver NeK5000 [41] which is based on the spectral element method (SEM). A $\mathbb{P}_{N}-\mathbb{P}_{N-2}$ formulation has been used: the velocity field is discretised using $N$ th degree Lagrange interpolants, defined on the Gauss-Legendre-Lobatto quadrature points, as basis and trial functions, while the pressure field is discretised using Lagrange interpolants of degree $N-2$ defined on the Gauss-Legendre quadrature points. Finally, the time integration is performed using the BDF3/EXT3 scheme: integration of the viscous terms relies on the backward differentiation (BDF3), while the convective terms are integrated explicitly using a third order accurate extrapolation (EXT3), both with a third-order accuracy. We wish to compare our forcing method with some benchmark, deterministic transition scenario, namely the K-type transition [37]. Therefore we set our computational box dimensions as $\left[L_{x}, L_{y}, L_{z}\right]=[2 \pi / 1.12,2,2 \pi / 2.10]$, as well as the numerical resolution to $N_{x} \times N_{y} \times N_{z}=128 \times 128 \times 128$ gridpoints on a $16 \times 16 \times 16$ spectral element grid with spectral order equal to 8 . We have verified that the chosen parameters of the numerical discretization are sufficient to accurately reproduce the dynamics of perturbations in a channel flow $[24,23]$. The Reynolds number is set to a subcritical value, namely $R e=5000$; this value is kept constant in all the numerical simulations discussed in this work. As already mentioned, periodicity is enforced in the streamwise and spanwise directions while a Dirichlet boundary condition is imposed at wall normal boundaries.

\subsection{Transient dynamics}

In this section we test whether the $F$ - type forcing method is capable of delivering a velocity perturbation (within a linear approximation) attaining the target $T u^{\text {linear }}$. The initial condition is set to $\boldsymbol{u}(\boldsymbol{x}, t=0)=\mathbf{0}$, and the synthetic volume forcing constructed using equation (22) is injected into equation (3). In Fig. 5 one can observe the time evolution of the perturbation energy, which increases from zero up to the prescribed target value, regardless of the number of frequencies used to discretize the energy spectrum, $N_{\omega}$. The asymptotic behaviour, related to the particular solution, is attained as long as the homogeneous solution is damped out, see equation (6), for a time $t \rightarrow \infty$. To characterize the transient behaviour we define the Rising Time $(R T)$ so that:

$$
\left|\frac{T u(R T)-T u^{\text {linear }}}{T u^{\text {linear }}}\right| \leq \epsilon,
$$

where $\epsilon$ is arbitrarily small; in the present work $\epsilon=10^{-3}$. As shown in Fig. 5, $R T$ increases with $N_{\omega}$, being approximately proportional to the fundamental frequency of the whole forcing signal. It is worth noting that, while for $N_{\omega}=1$ we have $R T \propto 2 \pi / \omega$, when increasing $N_{\omega}$ the rising time rapidly becomes inconveniently long [42]. In order to remove this transient behaviour we set as initial condition the velocity perturbation resulting from equation (8) for the previously constructed synthetic forcing, imposing both $\boldsymbol{u}$ and $\boldsymbol{f}$ with the associated time phases predicted by the resolvent analysis. In this way, we are able to cancel out the rising time, as shown in Fig. 6 , attaining the desired $T u^{\text {linear }}$ already at $t=0$. From now onward all our simulations are initialised as shown in this section.

\subsection{Recovering the optimal dynamics}

To further test our numerical setup we verify whether our fully 3D simulation is able to recover the 1D behaviour predicted by the optimal forcing analysis. Thus, we construct a forcing whose energy is concentrated on only one given couple of spatial wavenumbers, namely $\alpha=1.12, \beta=0.0\left(N_{\alpha, \beta}=1\right)$, and 8 temporal frequencies $\left(N_{\omega}=8\right)$. The energy spectrum of the input signal (squares), as well as that of the output perturbation as provided by the numerical simulation (circles) and by the resolvent analysis (diamonds) are depicted in Fig. 7. The system energy response $\|\boldsymbol{u}\|_{E}^{2}$ matches the theoretical one $R(\omega)\|\boldsymbol{f}\|_{E}^{2}$ within the forcing range $\left[\omega_{\min }, \omega_{\max }\right]$. Conversely, for higher frequencies the energy level predicted by the 


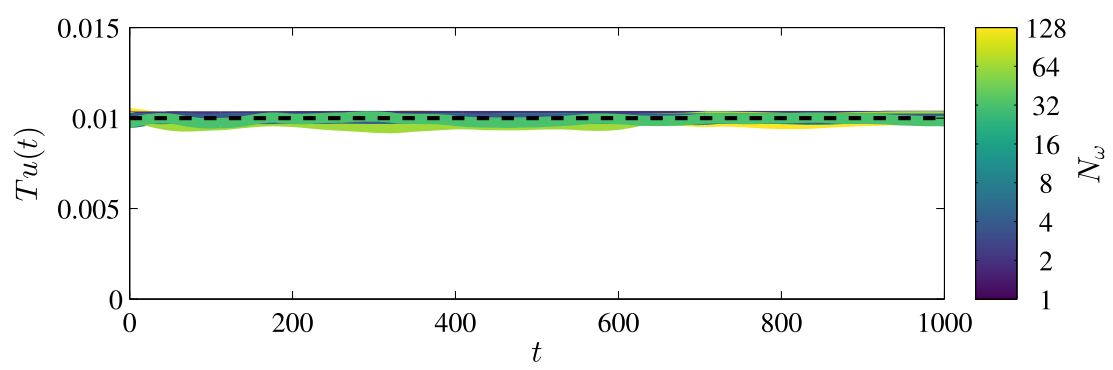

Fig. 6. Same as in Fig. 5, but with $\boldsymbol{u}(\boldsymbol{x}, t=0) \neq 0$, as computed with equation 23. Differently from Fig. 5, the flow immediately settles to the asymptotic regime, without any rising time.

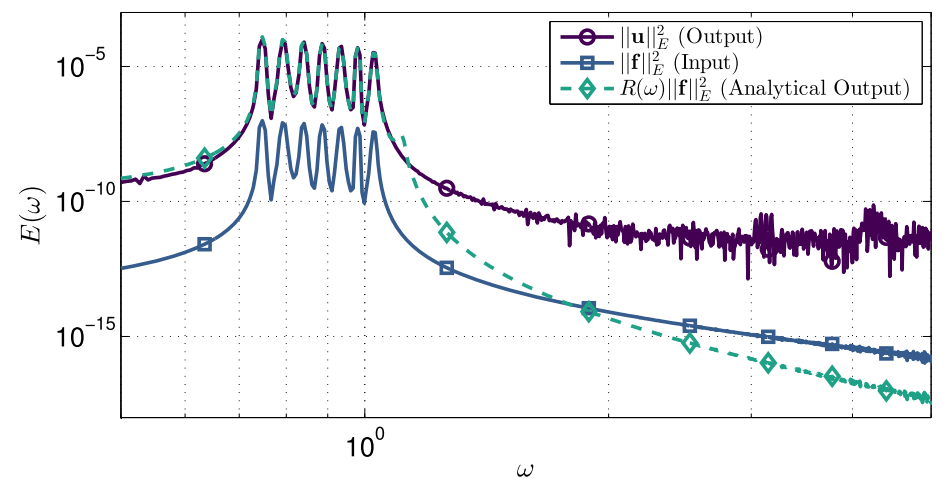

Fig. 7. Energy spectra of the forcing (blue solid line) and associated response obtained by DNS (purple solid line) and by the resolvent analysis (dashed line) with $N_{\alpha, \beta}=1,(\alpha=1.12, \beta=0.0), N_{\omega}=8$, and $\left[\omega_{\min }, \omega_{\max }\right] \approx[0.747,1.120]$.

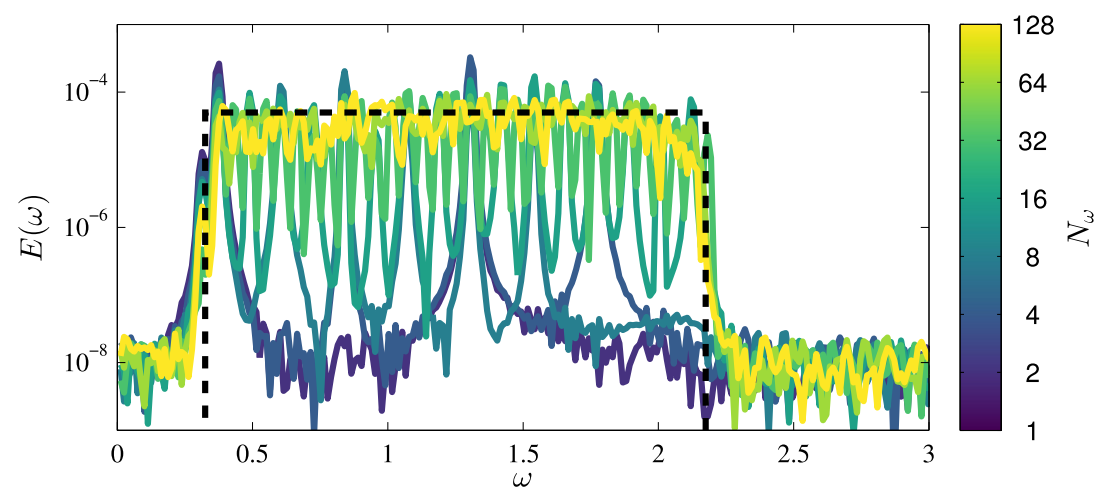

Fig. 8. Energy spectra extracted from several DNS using different values of $N_{\omega}$. The dashed line represents the target energy spectra prescribed for constructing the F-type forcing.

resolvent analysis is well below the threshold observable with our DNS code, which is affected by numerical noise. One can also notice the large energy gap between $\|\boldsymbol{u}\|_{E}^{2}$ and $\|\boldsymbol{f}\|_{E}^{2}$ : this is due to the optimal shape of the forcing for each given frequency, providing high values of the resolvent norm $R$. Thus, the F-type forcing method provides, within a linearized framework, the lowest possible input forcing amplitude for a given target output energy.

\subsection{Spectrum analysis}

In this subsection we investigate the influence of $N_{\omega}$ on the energy spectrum recovered through DNS with F-type forcing.

In Fig. 8 one can observe that the shape of the spectra deeply changes with $N_{\omega}$, becoming less spiky when this parameter is increased. Moreover, the area below the curve $E(\omega)$ must be the same for all the simulations, being $T u^{\text {linear }}$ constant. This results in a flattening of the energy spectra for $N_{\omega} \rightarrow \infty$. In particular, it appears that the energy spectra become almost independent from $N_{\omega}$ when more than 64 forcing modes are used. Thus, we set $N_{\alpha, \beta}=4, N_{\omega}=64$ throughout the reminder of the present work.

As a further validation of the method, we have imposed a different target energy spectrum, namely that extracted from DNS of a fully turbulent channel flow. First, we have recovered the turbulent energy spectrum at $R e_{\tau}=210$ (see purple line 


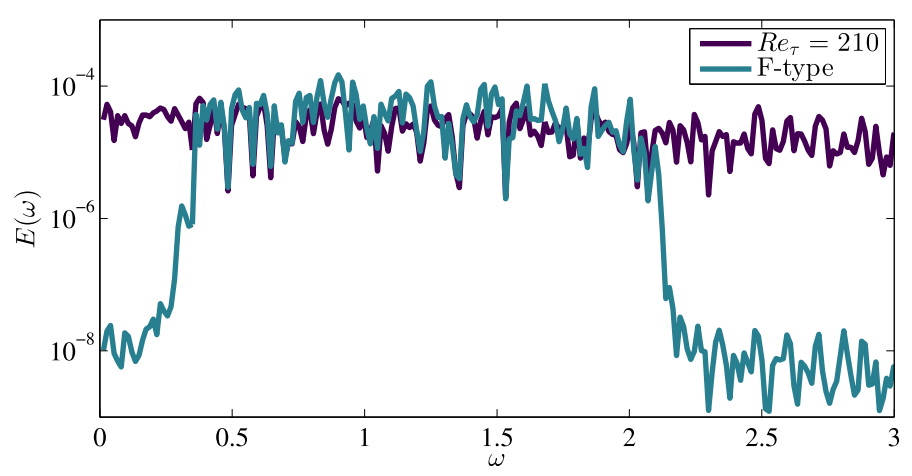

Fig. 9. Energy spectra extracted from a fully developed turbulent channel flow $R e_{\tau}=210$, used as target energy spectra for constructing the F-type forcing. The method, that for this test has been constrained within a forcing range $\left[\omega_{\min }, \omega_{\max }\right]=[1 / 3,2]$ for sake of visualisation, can accurately mimic the signature of a turbulent flow. Here we have used $N_{\omega}=64$ and $N_{\alpha, \beta}=4$.

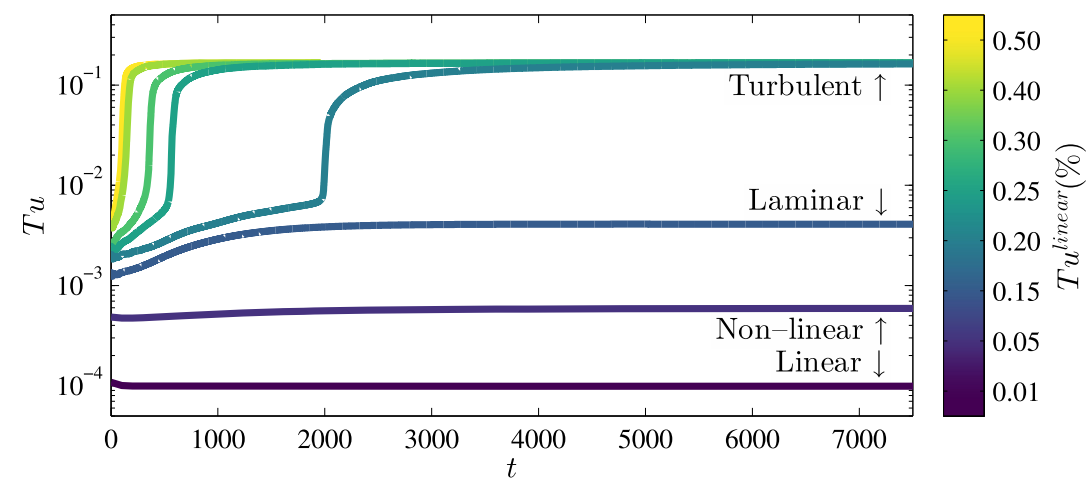

Fig. 10. Time evolution of $T u$ measured in different nonlinear DNS where the F-type forcing has been imposed at different target energies $T u^{\text {linear }}$ indicated in the legend.

in Fig. 9), resulting from turbulent transition starting from the laminar flow at $R e=5000$. Then, we have set it as a target spectrum in a further computation where the F-type volume forcing is imposed, in order to show that our method is capable of reproducing a stochastic volume forcing with any given spectrum. This is clearly demonstrated by the light blue curve, which follows closely the purple one representing the prescribed turbulent spectrum, within the selected frequency range. Please notice that the energy associated to each frequency recovered in a fully turbulent channel flow remains close to the mean value independently of the frequency, justifying our initial choice to use a flat energy spectrum. In fact, a flat energy spectrum would be recovered by ensemble-averaging several realisations of the turbulent flow in the same conditions.

\section{Non-linear dynamics}

In the previous section we have shown how the $F$ - type forcing method is capable of providing, within the linear framework, a synthetic noisy velocity perturbation with prescribed $T u^{\text {linear }}$. However, it is still to be verified whether the provided method is capable of triggering transition for a given target energy level, eventually leading to sustained turbulence. Aiming at a qualitative and quantitative comparison of our non-linear results with the literature, we set Re and the domain size $\left(L_{x}=2 \pi / \alpha, L_{z}=2 \pi / \beta, \alpha=1.12, \beta=2.10\right)$ in order to match the well known $K-t y p e$ channel-flow transition case [43,24,24], that is commonly used as benchmark in numerous numerical studies of turbulent transition [37,25,44,45]. From now onward we will show results derived from Direct Numerical Simulation of the fully non-linear Navier-Stokes equations, all computed for $N_{\omega}=64, N_{\alpha, \beta}=4$, using the procedure summarised in Algorithm 1 , using the same Re and numerical discretisation applied in section 3.

\subsection{Evolution of the turbulence intensity}

Once the domain size and the parameters $N_{\omega}$ and $N_{\alpha, \beta}$ have been selected, the only physical control parameter that remains to be set is the intensity of the energy perturbation field generated by the forcing perturbation, which coincides with $T u^{\text {linear }}$ in the linear regime. Fig. 10 shows the evolution of $T u$ measured in 6 direct numerical simulations (solid lines) forced using the F-type method with different target energy levels $T u^{\text {linear }}$. For low levels of $T u^{\text {linear }}$, the system response to the forcing matches the linear prediction as expected ( $T u^{\text {linear }}$ remains equal to the imposed level $T u=10^{-4}$ ), as shown by the bottom line designated as linear. Increasing $T u^{\text {linear }}$ we observe that the perturbation energy slowly increases starting 


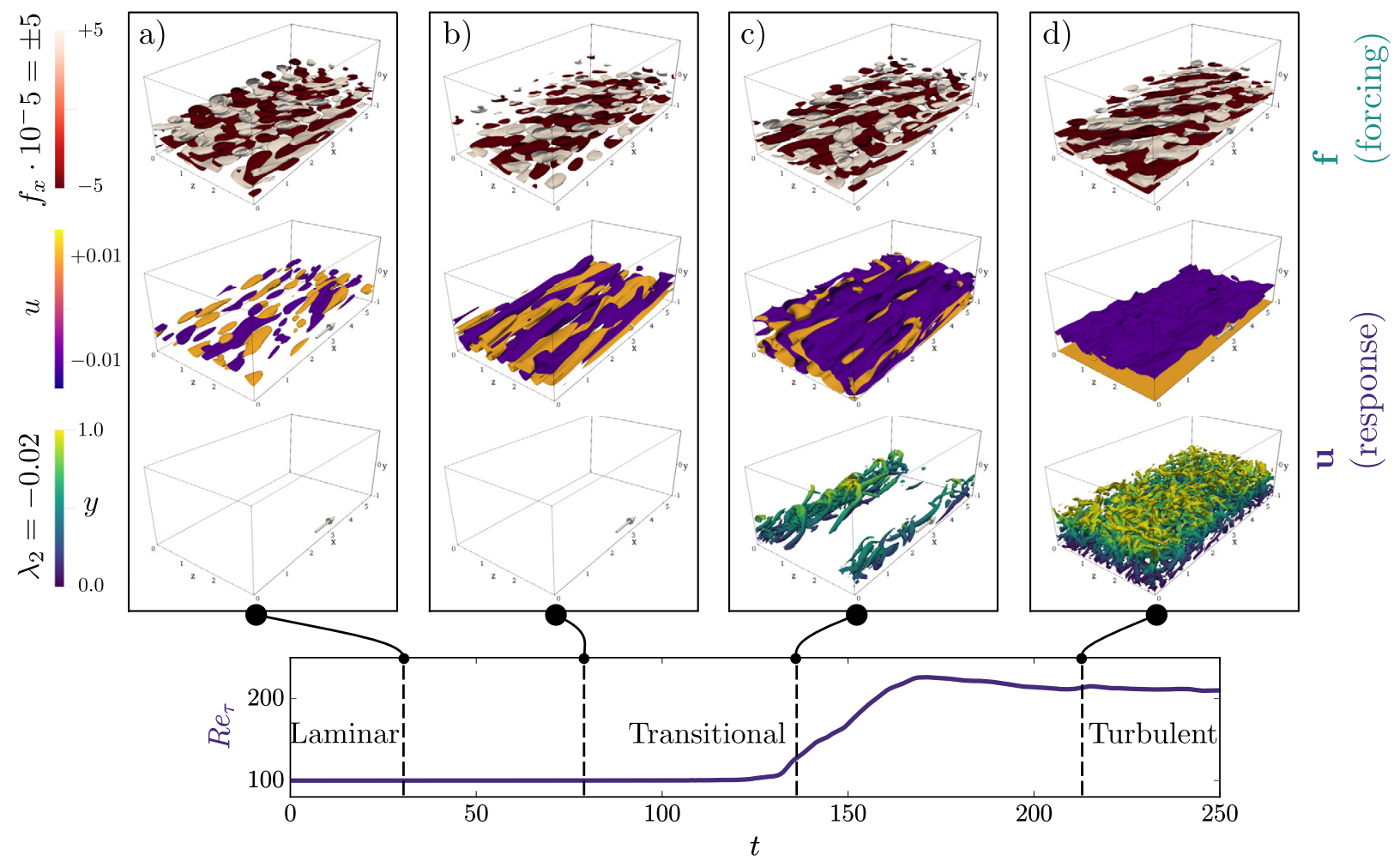

Fig. 11. Uncontrolled transition scenario obtained with the F-type method. Boxes a), b), c) and d) depict the isosurfaces of the streamwise component of the forcing (top), the streamwise velocity perturbation $u$ (middle) as well as the isosurfaces of the $\lambda_{2}$ criterion to visualise the onset of coherent vortical structures. The snapshots are extracted from a DNS of a 3D transitional channel flow with $T u^{\text {linear }}=0.5 \%, N_{\omega}=64, N_{\alpha, \beta}=4$, for $t=25,75,130,215$, respectively; the lower plot represents the friction Reynolds number $R e_{\tau}$ (see equation (29)), which indicates whether transition has taken place. Only the lower half of the channel is displayed for sake of clarity.

from the imposed level $T u^{\text {linear }}$, reaching asymptotic saturation. This phenomenon is due to the fact that the prescribed forcing energy level is high enough to trigger non-linear effects, even if the flow still remains in the laminar regime. Further increasing $T u^{\text {linear }}$ one can observe, after an initial slow increase of $T u$ due to the previously mentioned nonlinear laminar phase, a sharp jump in $T u$ due to laminar-turbulent transition. Thus, the transition threshold for an initial $T u^{\text {linear }}$ sits between the two lines designated as laminar and turbulent, for the prescribed value of the Reynolds number. Increasing the energy of the forcing beyond the transition level one can observe that the nonlinear laminar phase shrinks in time, leading to transition in lower simulation time. It is worth to notice that, regardless of the initial energy level, the final $T u$ for the statistically converged turbulent cases remains the same. The transition scenario obtained for the case with $T u^{\text {linear }}=0.5 \%$ (corresponding to the yellow line in Fig. 10) is depicted in Fig. 11. The streamwise component of the forcing (top) and of the resulting response (middle) as well as the response $\lambda_{2}$ surfaces (bottom) are provided at $t=25,75,130,215$ along with the $R e_{\tau}$ curve (see equation (29)) allowing to identify the different stages of transition. At first, the velocity perturbations resulting from the receptivity of the flow to the volume forcing are localized in the flow bulk (see box (a) of the figure). This bulk noise then penetrates close to the wall through receptivity triggering elongated streamwise velocity perturbations (box (b)). A fully non-linear transitional phase is reached when these streaky structures become unstable generating vortices on their top and flanks, among which some hairpin-like vortices can be recognized in box (c). Finally, after breakdown to turbulence, the flow becomes statistically homogeneous in both spanwise and streamwise direction (see box (d)).

\subsection{Wavenumber decomposition}

A powerful tool to study and unveil the mechanisms occurring in the first stages of transition is to analyse the mode-bymode energy evolution, performing a spatial Fourier decomposition of the flow field at each timestep [23,27]. The results of this procedure are shown in Fig. 12, where the different spatial modes are identified by the couple of integers $\left(i_{\alpha}, i_{\beta}\right)$ indicating the $i$ th multiples of the fundamental wavenumbers $2 \pi / L_{x}, 2 \pi / L_{z}$ (see also [27]).

For high enough $T u$ levels, streamwise invariant streaky structures (identified by modes $\left(0, i_{\beta}\right)$ rapidly appear and grow in amplitude, despite not being injected in the flow via the synthetic forcing/response, for the reasons discussed in section 2.3. This is probably due to the fact that the optimal perturbation [32] for shear flows has the form of a pair of streamwise streaks; thus, this kind of streamwise-invariant structure is also the most likely to occur when the laminar flow is perturbed, as discussed in Ref. [46]. For low values of $T u^{\text {linear }}$ (frame 12b) the streaky modes are characterized by an 


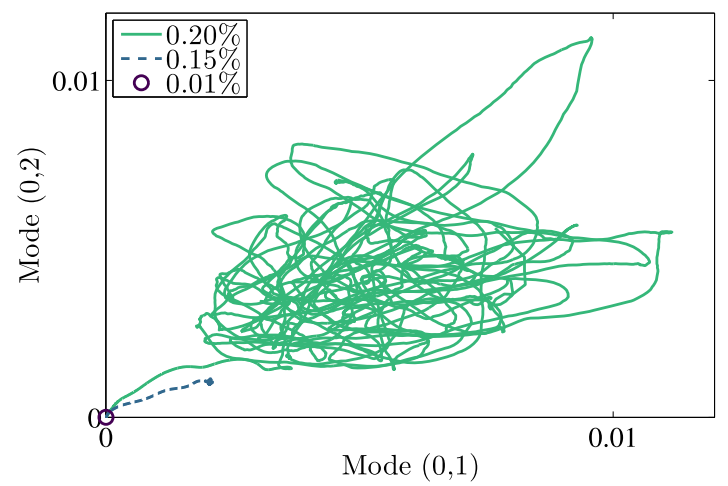

(a) Phase portrait for different $T u^{\text {linear }}$. Amplitude of the first two streamwise invariant Fourier components.

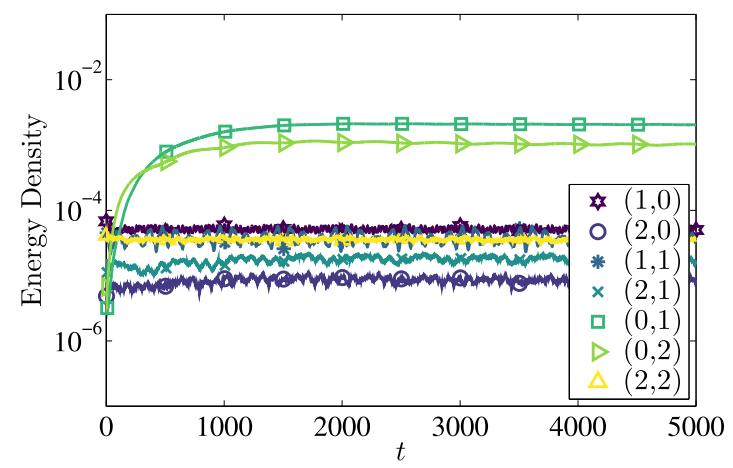

(c) Time evolution of the energy density of different Fourier modes for $T u^{\text {linear }}=0.15 \%$

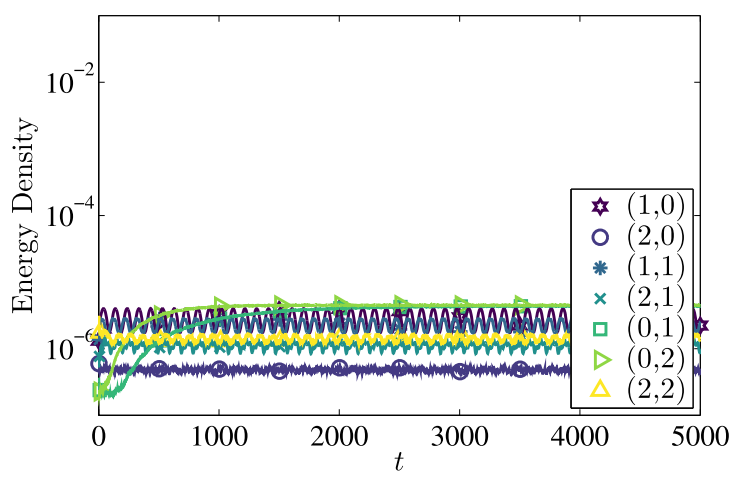

(b) Time evolution of the energy density of different Fourier modes for $T u^{\text {linear }}=0.01 \%$

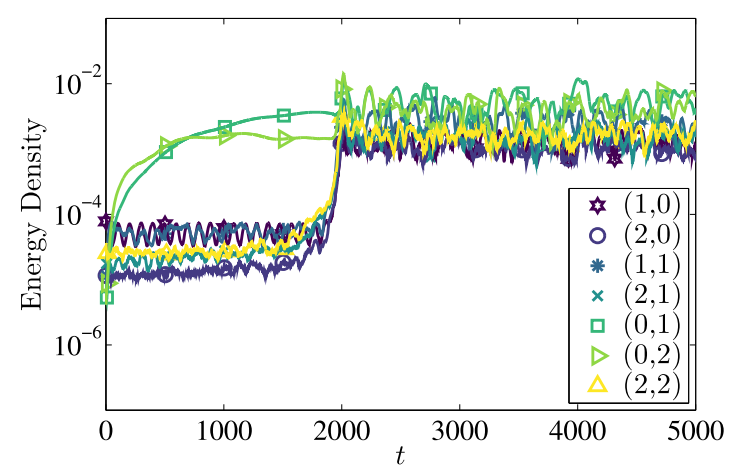

(d) Time evolution of the energy density of different Fourier modes for $T u^{\text {linear }}=0.20 \%$

Fig. 12. Energy density of selected Fourier components $\left(i_{\alpha}, i_{\beta}\right)$ for different values of $T u^{\text {linear }}$. Subframe (a) is a phase portrait representing the evolution of the first two streamwise-invariant modes generated by the non-linear interactions, usually referred as streaks [31], for different values of $T u^{\text {linear }}$. Subframes (b, c, d) show the emergence of the streaky modes from the forced background noise. Depending on the forcing energy levels, several flow behaviours are observed, from laminar to turbulent (see Fig. 10).

amplitude comparable with that of the other modes, and of the linear forcing itself. Increasing the value of $T u^{\text {linear }}$ (frame 12c) we observe a strong increase of their amplitude leading to nonlinear saturation, despite the flow remaining laminar, as discussed before. For the largest value of $T u^{\text {linear }}$ (frame $12 \mathrm{~d}$ ) secondary instability of the streaks is triggered after nonlinear saturation, leading to turbulence. A similar behaviour has been observed in Ref. [27] when triggering transition by using noise (constructed as a sum of random Stokes modes) as initial condition in a temporal channel flow, as provided in Fig. 13 (left frame). Comparing these literature results with those obtained by the F-type forcing method for high $T u^{\text {linear }}$ values (right frame), one can observe that the time evolution of the energy density of the Fourier components is similar, although some discrepancies can be found. For instance, at the very beginning of the F-type transition process, modes $(1,0)$, $(1,1)$, and $(0,1)$ strongly increase their energy due to the high receptivity of the flow to the imposed forcing. Whereas, when random Stokes modes are used, an initial decrease of these modes energy is observed, followed by an energy increase of the streaky modes only. This notable difference in the initial phases of transition can be better visualized in Fig. 14, showing the early time evolution of $T u$ in both cases. When the flow is fed with random noise, a non-physical initial transient is observed, where $T u$ initially decreases while the disturbance is adapting itself to the underlying Navier-Stokes equations, then steeply increases again, saturates, and begin to substantially grow (mostly due to non-linearity) only at $t \approx 60$. Whereas, when the F-type method is used, $T u$ increases algebraically already at the very beginning of the simulation, since the flow receptivity is exploited optimally to trigger energy growth. This allows to induce transition with lower amplitude perturbations as compared to random noise.

\subsection{Transition energy thresholds}

As the transition location in a spatially-evolving flow is strongly influenced by the intensity of the incoming perturbed flow [37], in a streamwise periodic flow the transition time is directly affected by the chosen $T u^{\text {linear }}$. To identify transition to turbulence we measure the Reynolds number based on the friction velocity: 

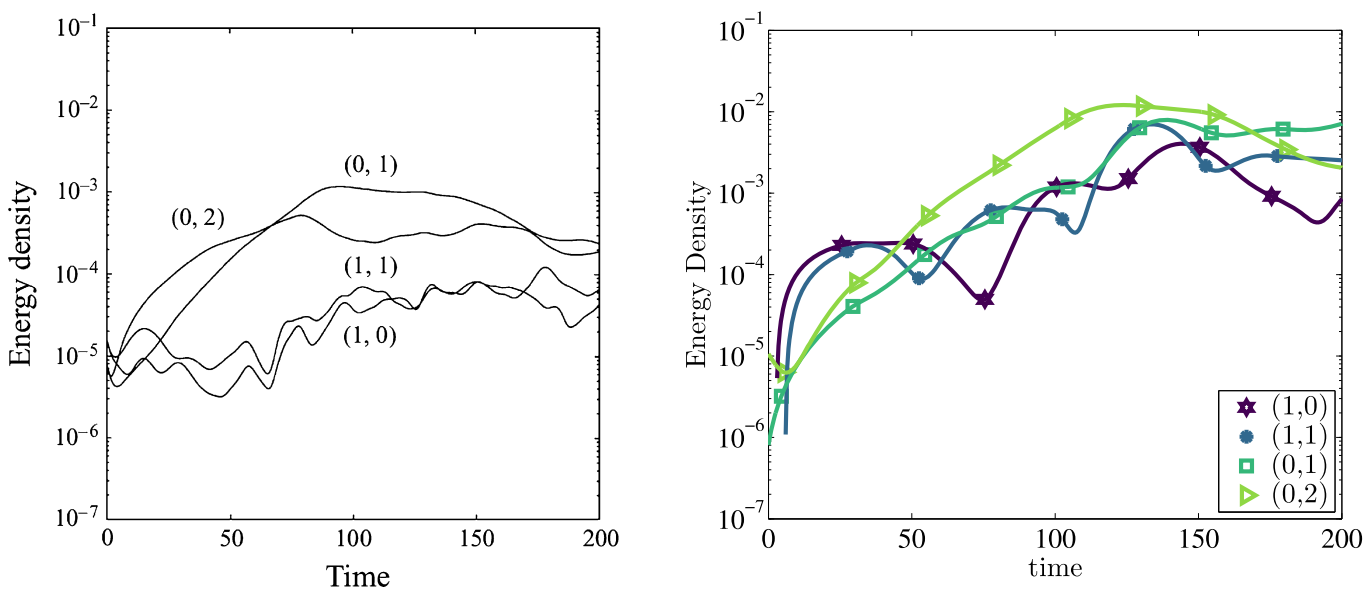

Fig. 13. Energy density of selected Fourier modes $\left(i_{\alpha}, i_{\beta}\right)$ for a DNS with initial condition composed by random Stokes modes (N-type transition) as computed by [27] (left frame) and the F-type forcing method (right frame) both for $R e=5000$. The energy of the initial perturbation for the N-type transition is $\|\boldsymbol{u}(t=0)\|_{E} \approx 2 e^{-4}$. The forcing intensity for the F-type method has been set so that the induced velocity field attains the same energy value. Even though the velocity fields induced by the F-type method are initially devoid of $\alpha=0$ modes, these modes rapidly grow in amplitude overtaking the other ones, as also observed in the N-type transition.

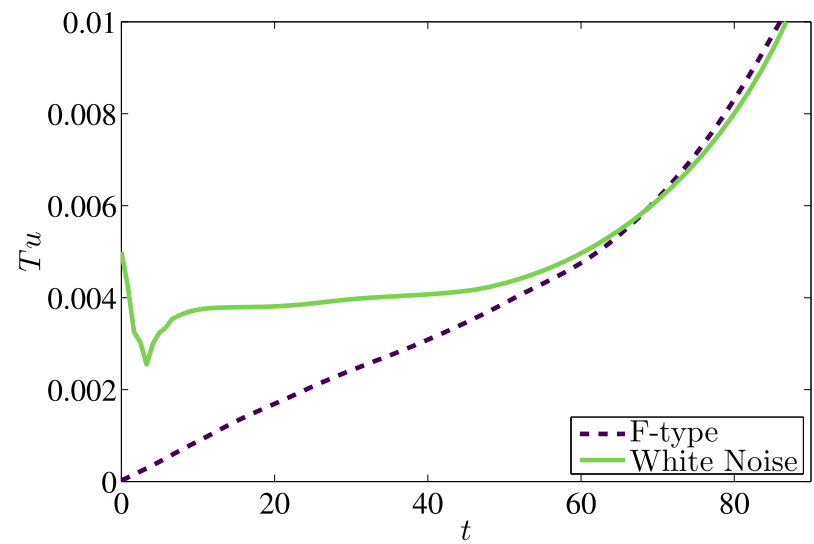

Fig. 14. Time evolution of $T u$ during the initial phases of transition for the F-type method and for white noise perturbations. $T u^{\text {linear }}=0.05 \%$ is used for the F-type method, while the initial random noise has been scaled in order to reach $T u(t=0)=0.05 \%$ as well. The $T u$ growth in the initial phase is almost linear for the F-type method.

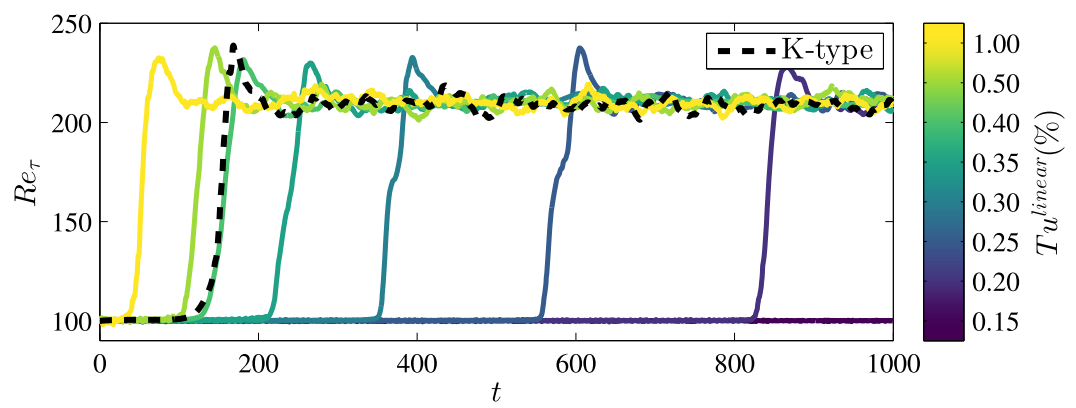

Fig. 15. Time evolution of $R e_{\tau}$ for the standard K-type transition scenario [21] (dashed line) and the F-type forcing method for several forcing levels (continuous lines coloured by the imposed $T u^{\text {linear }}$ value).

$$
\operatorname{Re}_{\tau}=\sqrt{\operatorname{Re}\left|\frac{\partial\langle u\rangle}{\partial y}\right|_{\text {wall }}},
$$

where $\langle\cdot\rangle$ represents the spatial average in the two homogeneous directions at a given time. The time evolution of $\operatorname{Re}_{\tau}$ for several values of $T u^{\text {linear }}$ is shown in Fig. 15 by the continuous lines, compared to the standard K-type transition scenario 


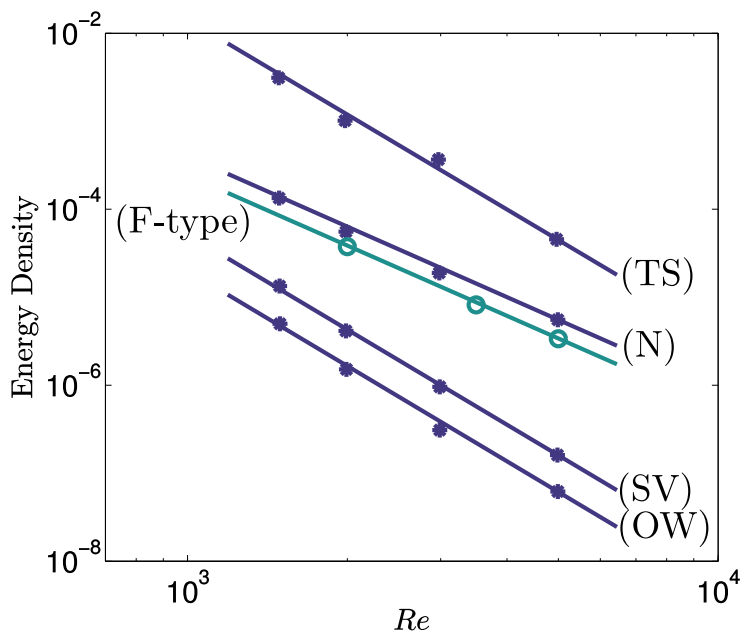

Fig. 16. Threshold energy densities for triggering transition using the synthetic forcing method proposed here (F-type) and for several transition scenarios [27] where different velocity fields are used as initial condition, namely Tollmien-Schlichting waves (TS), random noise (N), streamwise vortices (SV) and oblique waves (OW).

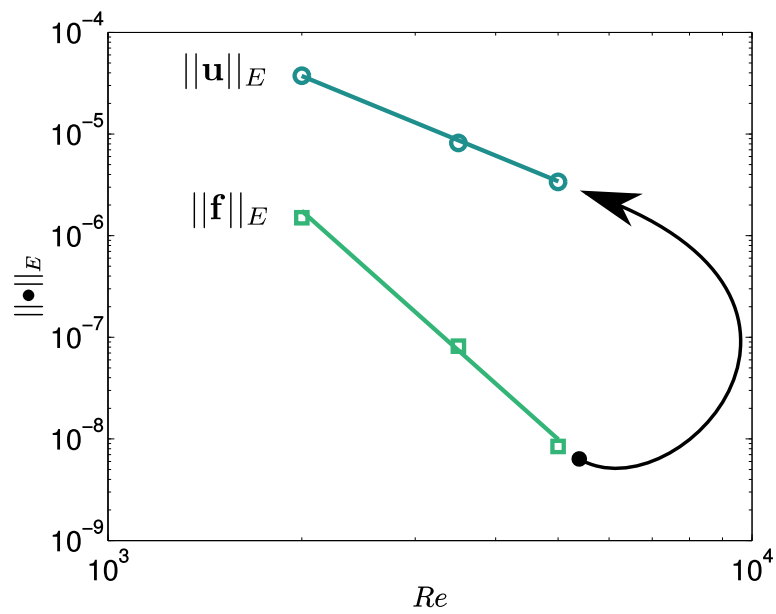

Fig. 17. Threshold energy densities for triggering transition of the imposed forcing as well as the resulting velocity perturbations, $\|\boldsymbol{f}\|_{E}$ and $\|\boldsymbol{u}\|_{E}$, whose amplitude is scaled by the resolvent norm $R$. The slope of the $\|\boldsymbol{u}\|_{E}$ and $\|\boldsymbol{f}\|_{E}$ lines differs since the resolvent norm $R$ increases with $R e$.

[21] (dashed line). Comparing Fig. 10 with 15 one can observe that $R_{\tau}$ strongly overtakes the initial laminar value only when turbulence is attained, confirming that its value can be used to detect when transition has taken place. Thus, Fig. 15 shows that the transition time decreases monotonically with the forcing energy $T u^{\text {linear }}$, similarly to the transition position in boundary layer flows [14]. Therefore, it appears that using the F-type forcing method one can easily control the transition time changing the control parameter $T u^{\text {linear }}$.

It is also worth to investigate the threshold energy able to trigger turbulence using the F-type method, as compared to other transition scenarios. The threshold energy density for different transition scenarios in channel flow is provided in Fig. 16 for different values of $R e$. Following Ref. [27], we consider that transition has taken place when $R e_{\tau}(t)$ overtakes the value $1.01 \operatorname{Re}_{\tau}$ (laminar). As one can observe in Fig. 16, the threshold energy density of the velocity perturbation field induced by the F-type forcing is comparable to that needed for triggering turbulence by using random perturbations (N-type scenario). However, there is a crucial difference between the F-type method and the N-type scenario. Fig. 17 provides the velocity perturbation energy $\|\boldsymbol{u}\|_{E}=\frac{1}{2 V} \int_{V} \boldsymbol{u}^{2}(\boldsymbol{x}, t) d V$ and its corresponding forcing norm $\|\boldsymbol{f}\|_{E}=\frac{1}{2 V} \int_{V} \boldsymbol{f}^{2}(\boldsymbol{x}, t) d V$. Since the flow is excited by a weighted sum of optimal forcings, the highest possible gain between the forcing itself and the induced velocity field is attained [32]. The curves in the figure show that the volume forcing energy injected within the flow is two to three orders of magnitude lower than the energy of the noisy velocity perturbation inducing transition. Thus, the F-type forcing method triggers transition to turbulence relying on a low-amplitude noisy volume forcing, without prescribing unrealistic initial perturbations linked to specific transition mechanisms (such as streamwise rolls, oblique waves, etc.) like the classical scenarios proposed in the literature. 


\section{Turbulent state}

In this section, the statistically converged turbulent state induced by the F-type forcing method with $T u^{\text {linear }}=0.5 \%$ is analysed, in comparison with that of the standard K-type transition method [37]. The fundamental wavenumbers $\alpha=$ $1.12, \beta=2.10$ are chosen, the dimensions of the numerical domain being $2 \pi / \alpha \times 2 \times 2 \pi / \beta$, discretized on a $N_{x} \times N_{y} \times N_{z}=$ $192^{3}$ grid. In Ref. [37], starting with Reynolds number $R e=(U H) / v=5000$ measured at the centreline, and keeping a constant flow rate throughout the whole simulation, a friction Reynolds number $\operatorname{Re}_{\tau} \approx 208$ is reached, which is defined using the friction velocity

$$
u_{\tau}=\sqrt{\frac{\tau_{\text {wall }}}{\rho}}
$$

with local shear stress at the wall given by:

$$
\tau_{\text {wall }}=\mu\left|\frac{\partial\langle u\rangle}{\partial y}\right|_{\text {wall }} .
$$

Using this value of the friction Reynolds number the chosen domain size and the grid resolution can be expressed in wall units as:

$$
\begin{aligned}
& {\left[L_{x}^{+}, L_{y}, L_{z}^{+}\right]=[\approx 1165,2, \approx 435]} \\
& {\left[\left.\Delta x^{+}\right|_{\text {mean }},\left.\Delta y^{+}\right|_{\text {wall }} /\left.\Delta y^{+}\right|_{\text {centre }},\left.\Delta z^{+}\right|_{\text {mean }}\right]=[7.3,0.040 / 4.1,3.9]}
\end{aligned}
$$

where the superscript + indicates adimensionalisation with respect to wall units, with $u_{\tau}=\operatorname{Re} \tau / \operatorname{Re} \approx 0.0417$ and $l^{+}=$ $1 / R e_{\tau} \approx 0.00481$. Thus, the selected computational domain is much larger than the minimal flow unit [35] and the grid size is sufficiently small to accurately describe a turbulent flow [37]. The time averages presented in the paper are based on a period of $T=500$ time units starting 500 time units after transition takes place.

Following [47], the average of the three-dimensional, time dependent field $f(x, y, z, t)$ in the homogeneous directions $x$ and $z$ reads:

$$
\bar{f}(y, t)=\frac{1}{L_{x} L_{z}} \int_{0}^{L_{x}} \int_{0}^{L_{z}} f(x, y, z, t) d x d z .
$$

The velocity field can be thus decomposed as:

$$
\mathbf{u}=\{\bar{U}(y, t), 0,0\}+\left\{u^{\prime}, v^{\prime}, w^{\prime}\right\}
$$

as well as the forcing field

$$
\mathbf{f}=\{\bar{F}(y, t), \bar{G}(y, t), \bar{H}(y, t)\}+\left\{f^{\prime}, g^{\prime}, h^{\prime}\right\} .
$$

It is important to note that in the F-type forcing method the spatial average of the forcing is strictly zero $(\{\bar{F}, \bar{G}, \bar{H}\} \equiv$ $\{0,0,0\})$, being constructed by a linear composition of Fourier modes in the homogeneous directions. Assuming $f$ to be statistically steady over $N$ periods of length $T$, we can define the phase average of $\bar{f}(y, t)$ as $\hat{f}(y, \tau)$, that can be written as:

$$
\hat{f}(y, \tau)=\frac{1}{N} \sum_{n=0}^{N-1} \bar{f}(y, n T+\tau) .
$$

The space-time average over a period $T$ is:

$$
\langle f\rangle(y)=\frac{1}{T} \int_{0}^{T} \hat{f}(y, t) d t
$$

and the global quantity $[f]_{g}$ is obtained integrating $\langle f\rangle(y)$ in the wall-normal direction:

$$
[f]_{g}=\int_{0}^{h}\langle f\rangle(y) d y .
$$




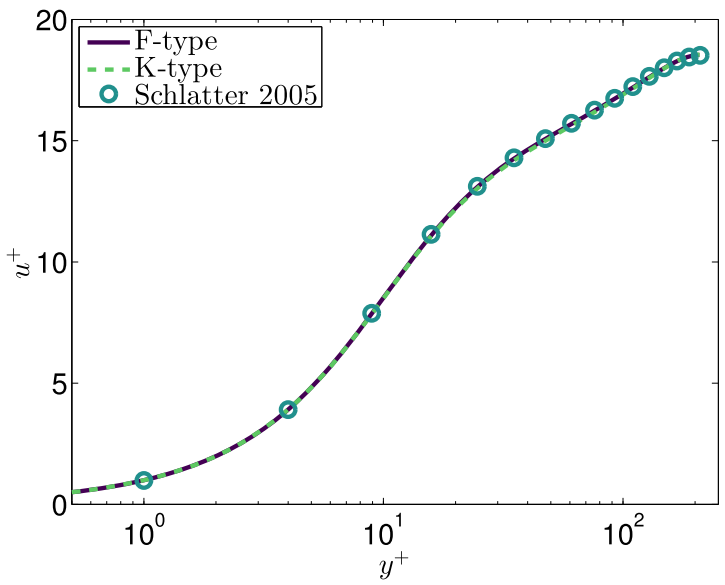

(a) Mean Velocity profiles $u^{+}=\langle u\rangle / u_{\tau}$

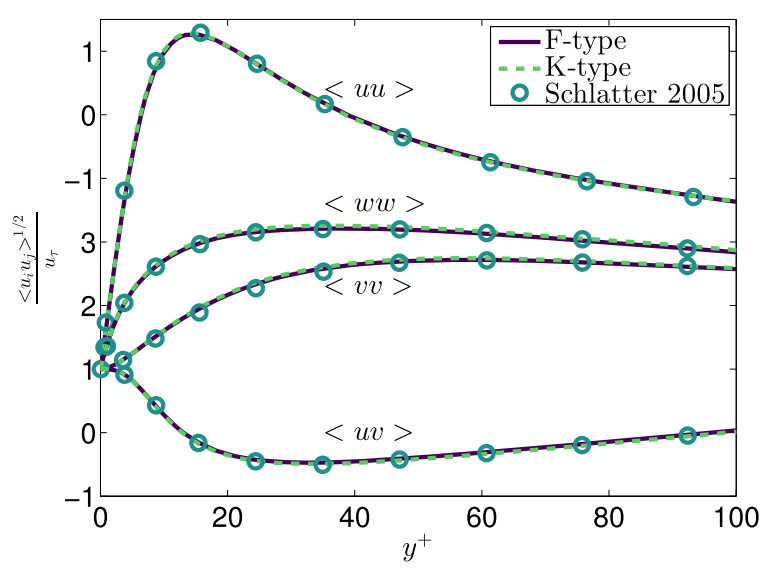

(b) Reynolds stresses

Fig. 18. Comparison of mean flow and Reynolds stresses extracted by fully turbulent DNS for a classical K-type [37] transition scenario as well as the F-type forcing method.

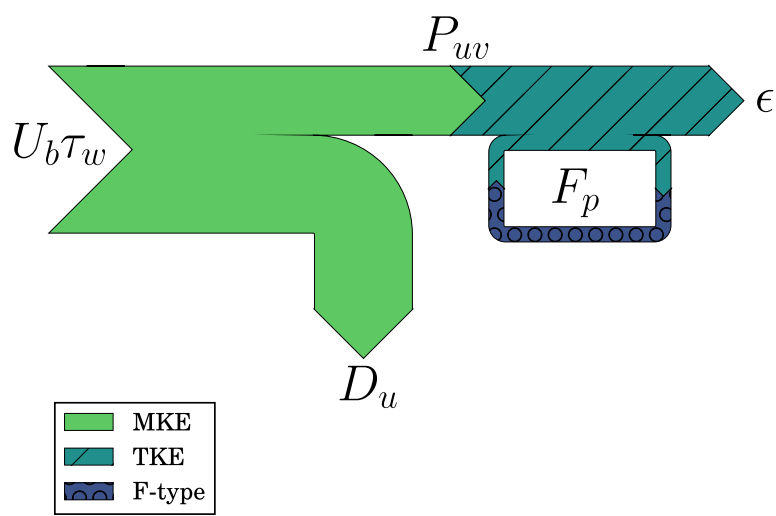

Fig. 19. Sketch of the global energy balance in a channel flow. Arrow widths are proportional to the corresponding values, except $F_{p}$ that is scaled by a factor of 1000 for the sake of visualization and exists only if a volume forcing is used. Energy is initially pumped into the flow via the $U_{b} \tau_{w}$ term. One part is dissipated by the mean flow $\left(D_{u}\right)$, the rest is transferred to the fluctuations via the production term of the turbulent kinetic energy $P_{u v}$, which eventually coincides with the turbulent dissipation $\epsilon$. The F-type method creates an additional sink-source energy $F_{p}$, but its effect turns out to be negligible.

\subsection{Statistics and energy budget}

Figs. 18a and 18b show the zero-th and first order statistics of fully turbulent DNS where transition has been triggered following different transition paths. As expected, the results of the different simulations are indistinguishable.

In order to determine whether the F-type forcing method affects in some way the energy balance within the turbulent flow, we analyse the transport equations of the Mean and Turbulent Kinetic Energy (MKE and TKE), identifying the terms associated with the volume forcing. The transport equations of the MKE and TKE are detailed in Appendix A, where it is also shown that the volume forcing affects the TKE equation only. A sketch of the energy budget in a statistically turbulent channel flow is provided in Fig. 19. In a fully turbulent channel flow, since both MKE and TKE are statistically zero, the energy pumped by the pressure gradient is transferred by the production term to the flow oscillations and dissipated by the mean flow as well as by the turbulent fluctuations. This standard scenario [5] is altered by the F-type volume forcing due to the introduction of an energy term linked to the forcing itself, $F_{p}$, whose form and derivation is detailed in Appendix A. In order to determine the amount of energy produced or dissipated by the volume forcing, we compute the time evolution of the different terms of the Kinetic Energy Budget (KEB), as in equation (A.14) in a statistically converged turbulent flow arising from different transition scenarios. The different terms are plotted in Fig. 20a, where one can observe that the term $F_{p}$ associated to the forcing in equations (A.15) is three to four order of magnitudes smaller than the other production or dissipation terms. Thus, this term can be considered negligible in the energy balance account. In fact, in Fig. 20a one cannot distinguish between the energy terms of the turbulent states obtained by the K-type and F-type methods, although in the latter the volume forcing remains active throughout the simulations. Fig. 20b provides a phase portrait describing the laminar-turbulent transition paths for the K-type and F-type methods. The time evolution of the production term $P_{u v}$ and 


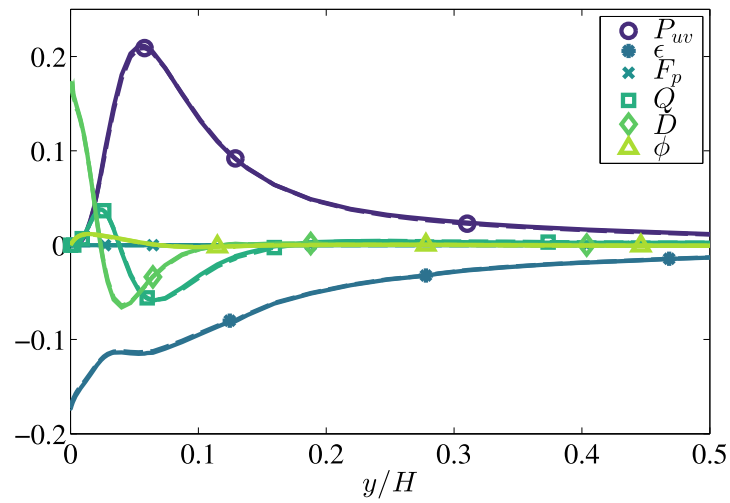

(a) Kinetic energy budget (A.14) for turbulent states triggered via K-type (solid line) and F-type (dashed line) transition methods.

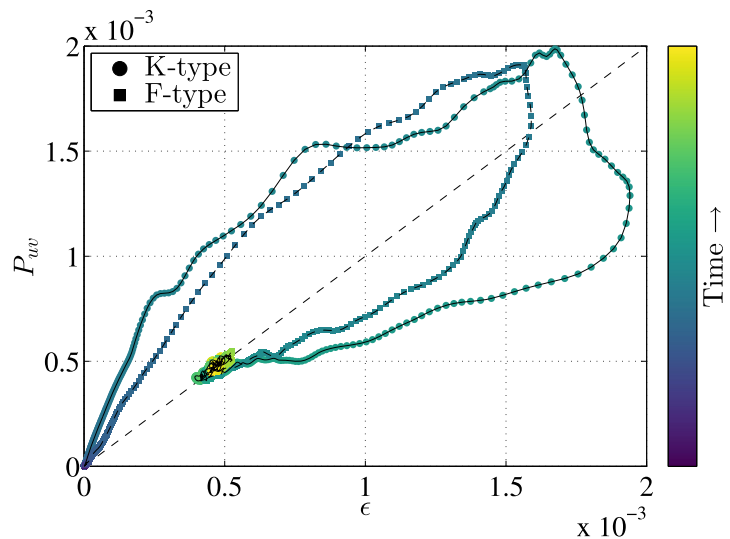

(b) Comparison of the time evolution of production $\left(P_{u v}\right)$ and turbulent dissipation $(\epsilon)$ terms during laminar-turbulent transition triggered with K-type and F-type methods.

Fig. 20. Turbulent Kinetic Energy Decomposition plots. Labels are defined in Appendix A.

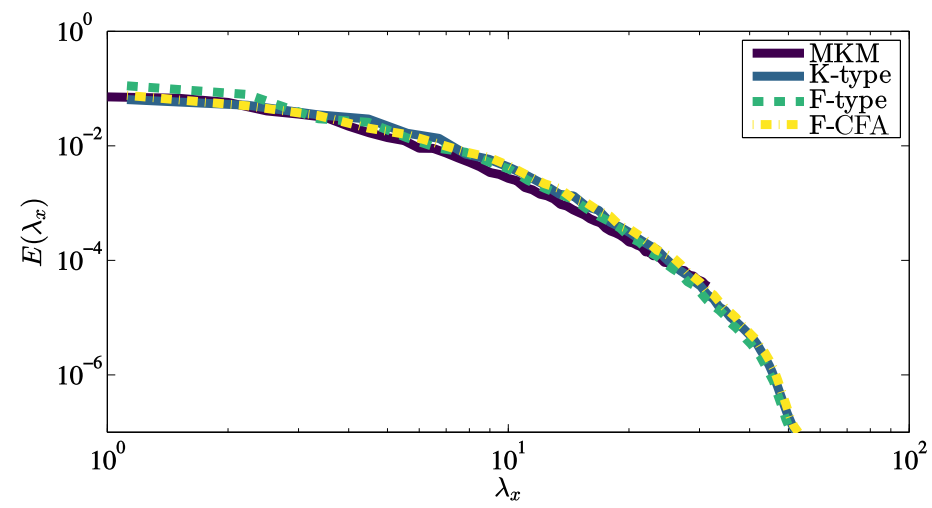

Fig. 21. Comparison of the streamwise velocity one-dimensional turbulent energy spectra $E_{u u}$ for $\Re_{\tau}=210$ at $y=0$ (midplane) for the K-type, F-type, and F-type with Controlled Forcing Amplitude (F-CFA) method, as discussed in section 5.3, as well as for the MKM benchmark literature case at $R e_{\tau}=180$ in Ref. [48]. The spectrum recovered with the F-type method slightly deviates from the expected result, as a result of the presence of the volume forcing. This behaviour can be avoided by simply switching the forcing off as explained in section 5.3, resulting in the F-type with Controlled Forcing Amplitude case. The slight misalignment between the spectra obtained from the present computations (K-type, F-type and F-CFA) and the reference case is due to the fact that the former are computed for $R e_{\tau}=210$, while the latter for $R e_{\tau}=180$.

the turbulent dissipation $\epsilon$ is very similar for both transition scenarios. Moreover, when the final turbulent state is reached, the energy budget terms perfectly match.

\subsection{Energy spectrum comparison}

To further analyse the turbulent flow we plot in Fig. 21 the one-dimensional energy spectra for the turbulent states triggered via the K-type and F-type transition, compared to the benchmark data extracted from Ref. [48]. The energy density associated with large wavenumbers is several order of magnitudes lower than that at low wavenumbers, confirming that the grid resolution is adequate for accurately describing a turbulent state. However, one can observe that, for low wavenumbers, the energy spectrum does not perfectly match the benchmark values when the F-type forcing method is used. In order to find a possible reason for this weak discrepancy we analyse the time variation of the friction Reynolds number, provided in Fig. 22. Comparing in Fig. 22a the $R e_{\tau}$ signals issued from the turbulent states obtained with the K-type and F-type methods, one can observe that the latter is characterised by higher frequency oscillations than the former. Performing a Fourier transform in the time range $500 \leq t \leq 1000$ and invoking Taylor's relation linking spatial and temporal frequencies, we have found that these high frequencies match with those imposed using the F-type volume forcing, as represented in Fig. 22b. To verify that the discrepancy of the spectra is indeed due to the imposed volume forcing, we suspend it once a fully turbulent state is obtained (for $t \geq 500$ in the considered case). In Fig. 22a the resulting $\operatorname{Re}_{\tau}$ signal is provided. The 


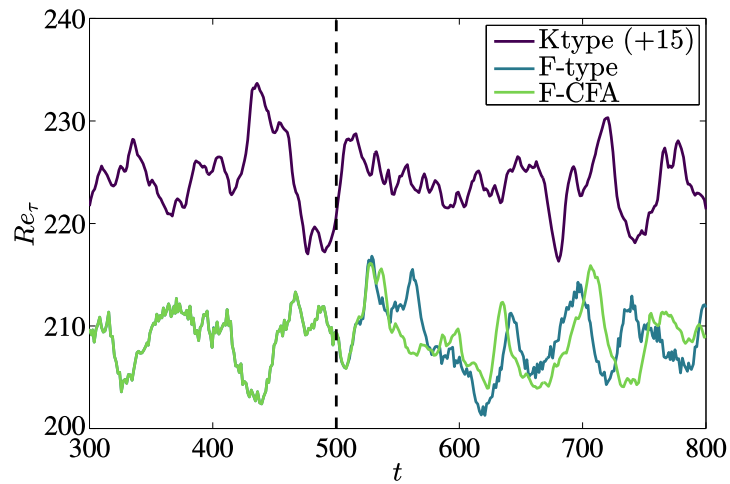

(a) $R e_{\tau}(t)$ signals. The vertical dashed line represents the time at which the forcing is interrupted.

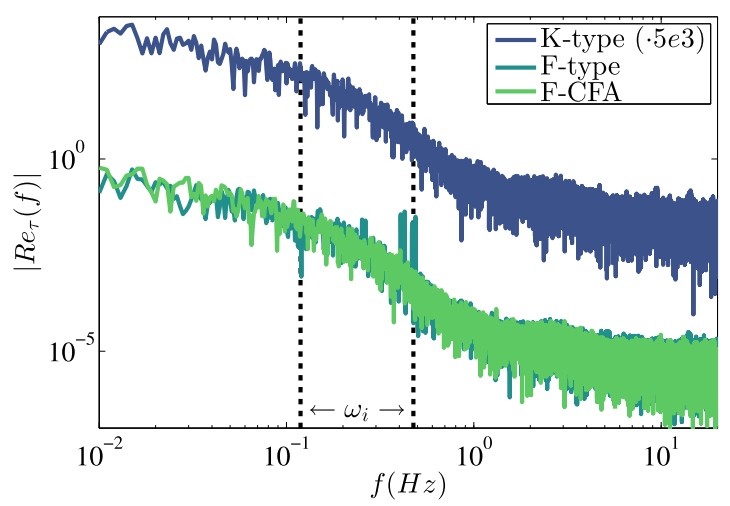

(b) $R e_{\tau}$ spectra for the signals presented on the left frame; $\omega_{i}$ indicate the forcing frequency range for the F-type method.

Fig. 22. $R e_{\tau}$ time signals and spectra for K-type (blue line), F-type (light blue line) and F-type with interrupted forcing (F-CFA, green line) methods. Spurious energy peaks in the F-type method occur within the same range of the forcing used to trigger transition, as indicated by the vertical lines in the right frame. If the forcing is turned off (for $t>500$ as indicated by the vertical line in the left frame), these spurious energy peaks disappear. K-type related data are shifted by a factor of 5000 for sake of readability.

high frequency oscillations rapidly decay when the forcing is interrupted, resulting in a spectrum devoid of spurious peaks, as shown in Fig. 22b. Finally, Fig. 21 shows that once turned off the forcing, the energy spectrum matches the benchmark one.

\subsection{Automatic forcing amplitude control}

We have shown in the previous subsection that the volume forcing needs to be turned off once the turbulent state is attained in order to avoid spurious frequencies in the energy spectrum. However, the time to reach a fully turbulent state depends on a number of factors, namely: the Reynolds number Re, the initial velocity disturbances shape and intensity (for K-type like methods), the $T u^{\text {linear }}$ forcing intensity (for F-type method), to cite a few. For this reason, in this section we aim at constructing a function capable of automatically adjust the forcing intensity without any a priori knowledge of the final state.

Towards this aim, we define two functions:

$$
A_{\text {shift }}(t)=\frac{\operatorname{Re}_{\tau}^{A}(t)-\operatorname{Re}_{\tau}(t)}{\operatorname{Re}_{\tau}^{A}(t)}
$$

and

$$
B_{\text {shift }}(t)=\frac{\operatorname{Re}_{\tau}^{B}-\operatorname{Re}_{\tau}(t)}{\operatorname{Re}_{\tau}^{B}}
$$

$\operatorname{Re}_{\tau}^{A}(t)$ being an exponential moving average (see Ref. [49]), computed at each iteration, it, as:

$$
\left[R e_{\tau}^{A}\right]^{i t}=\left[\operatorname{Re}_{\tau}\right]^{i t}+e^{(-d t \cdot T)} \cdot\left(\left[\operatorname{Re}_{\tau}^{A}\right]^{i t-1}-\left[\operatorname{Re}_{\tau}\right]^{i t}\right),
$$

where the prescribed averaging window is $T=100, d t$ is the time difference between the itth and the (it - 1)th iterations. Whereas, $R e_{\tau}^{B}$ is a function of the laminar Reynolds number only, namely, $\operatorname{Re}_{\tau}^{B}=\sqrt{2 \cdot \operatorname{Re}}$. Functions (39) and (40) have been constructed in order to show an opposed behaviour in the laminar and in the turbulent regime. In the laminar regime, being $\operatorname{Re}_{\tau}^{A} \approx \operatorname{Re}_{\tau}^{B}$, we have $A_{\text {shift }}(t) \approx B_{\text {shift }}(t)$; whereas, in the turbulent one, the difference $\operatorname{Re}_{\tau}^{B}-\operatorname{Re}_{\tau}$ will be much larger than $R e_{\tau}^{A}-R e_{\tau}$, resulting in $A_{\text {shift }}(t) \ll B_{\text {shift }}(t)$. Thus, for detecting transition to turbulence one can define the following function:

$$
\Delta_{F}=\left|\frac{A_{\text {shift }}}{B_{\text {shift }}}\right|
$$

which is used to gradually damp the F-type forcing once transition is triggered. Towards this aim, the forcing field issuing from the F-type forcing method is premultiplied by $\Delta_{F}$, which becomes equal to zero right after the flow reaches a selfsustained turbulent regime, as provided in Fig. 23. In the same figure one can observe the time evolution of $R e_{\tau}$ in the 


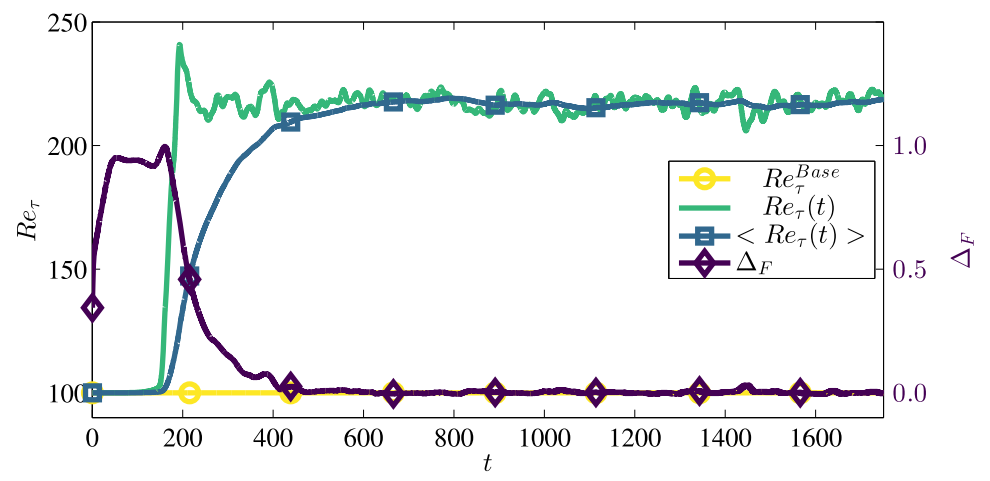

Fig. 23. Transition in a channel flow at $R e=5000$ using F-type method with Controlled Forcing Amplitude (F-CFA). The forcing amplitude $\Delta_{F}$ continuously decreases as the friction Reynolds number increases, finally reaching a zero value when $\operatorname{Re}_{\tau}$ attains the turbulent plateau.

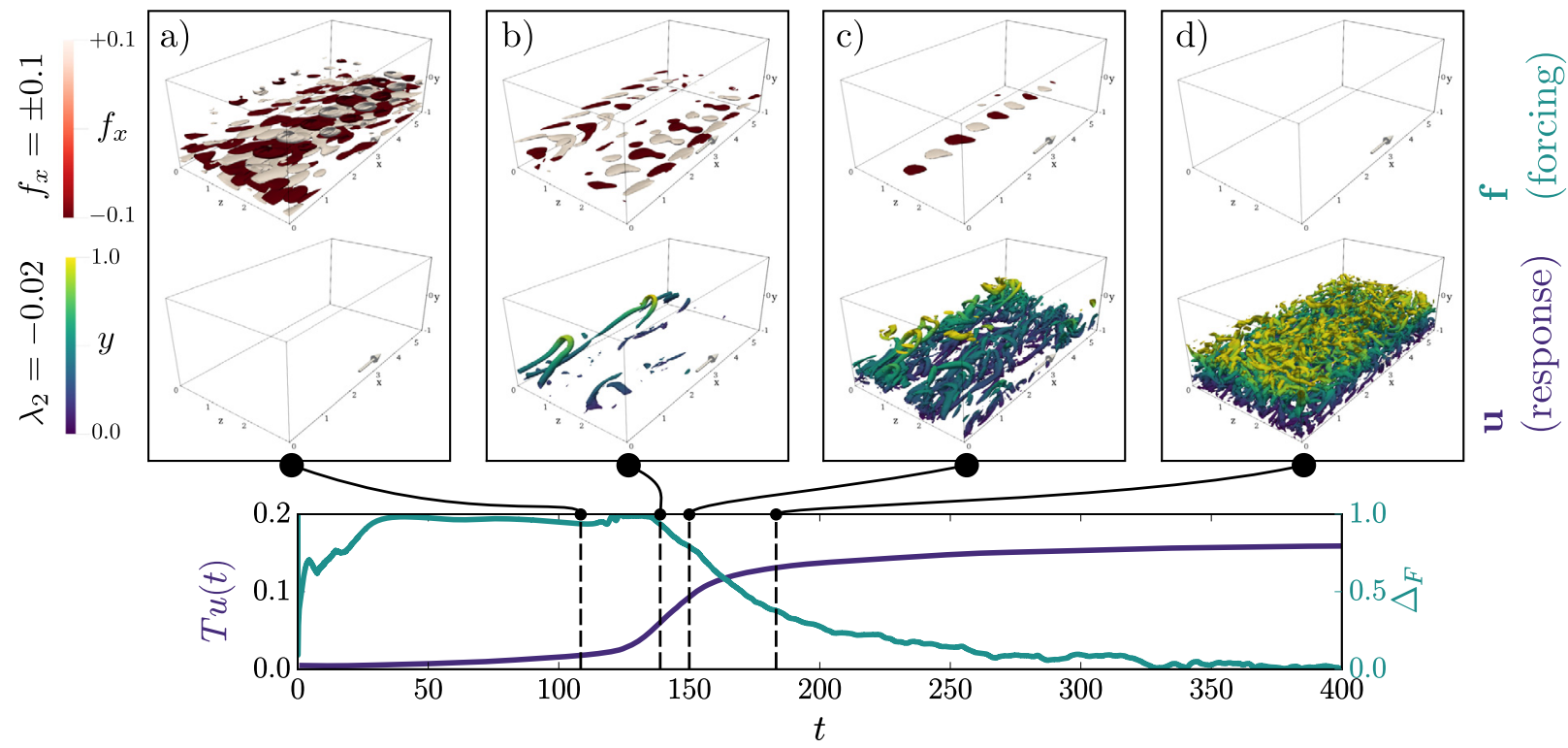

Fig. 24. Uncontrolled transition scenario, obtained with the F-type with Controlled Forcing Amplitude method. Boxes a), b), c) and d) depict the isosurfaces of the streamwise component of the forcing (top) and response (bottom) within the 3D transitional channel flow, for $t=110$, 140 , 150, 180 , respectively; the lower plot represents the turbulent intensity $T u$ as well as the automatic forcing amplitude control intensity $\Delta_{F}$ in time. It can be clearly seen that, when turbulent conditions are attained, the forcing shuts off automatically. In this computation $T u^{\text {linear }}=0.5 \%, N_{\omega}=64, N_{\alpha, \beta}=4$. Hairpin-like structures can be visualised in the $\lambda_{2}$-criterion surfaces during the late stages of transition, see b) and c). Only the lower half of the channel is displayed for sake of clarity.

Controlled Forcing Amplitude (CFA) case, together with its space-time average, showing the establishment of a fully turbulent flow. In pictures 23 and 25, we report the average value $\left[R e_{\tau}^{A}\right]^{i t}$ (referred to as $\left\langle R e_{\tau}(t)>\right.$ for the sake of notation), which appears to clearly indicate when transition is taking place, attaining a plateau when turbulence is finally achieved.

The transition scenario observed using the controlled amplitude F-type forcing with $T u^{\text {linear }}=0.5 \%$ is depicted in Fig. 24. The streamwise component of the forcing and the $\lambda_{2}$ surfaces of the associated response are provided at $t=110,140,150,180$. The stochastic forcing begins to fade away when the nonlinear phase of transition is attained. At $t=140$, a few isolated hairpin-like structures can be clearly observed, which rapidly create secondary hairpin vortices as well as small scale vortical structures (see the $\lambda_{2}$ surfaces in the bottom plots). At $t=180$ the turbulence intensity is saturating towards an asymptotic value, indicating that fully developed turbulence is almost attained. The forcing shuts off when $T u$ reaches its asymptotic value and turbulent conditions are attained.

We conclude this section by investigating whether the behaviour of the controlled amplitude parameter $\Delta_{F}$ is robust in a different flow setting. In fact, up to this point we have considered subcritical transition for $R e=5000$, as depicted in Fig. 24, close to the threshold for linear stability [31], but considerably larger than the minimum Reynolds number at which transition to turbulence can be observed in a channel flow [50], namely $R e \approx 1600$. For $R e<1600$, turbulence is not self-sustained [51]; thus, an initial perturbation superposed to the base flow might induce transition for a finite time, but the flow will eventually relaminarize [52]. Applying the F-type forcing method with controlled amplitude in such case, for instance at $R e=1250$, relaminarization is avoided since $\Delta_{F}$ never reaches zero, as shown in Fig. 25. 


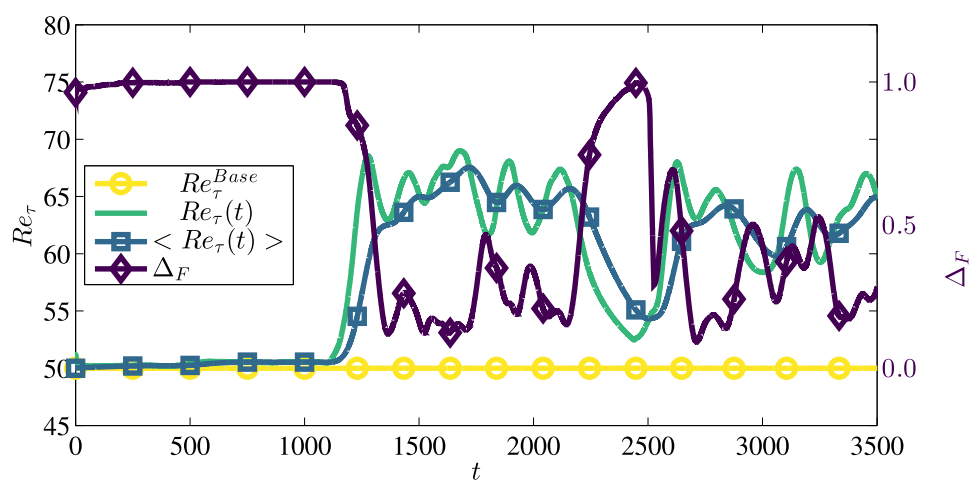

Fig. 25. Controlled amplitude F-type transition method for a low Reynolds number, $R e=1250$. The forcing intensity is the lowest one for which a transitional behaviour can be observed: $T u^{\text {linear }}=0.7 \%$. Forcing is activated intermittently to keep a turbulent state.

Whereas, by simply suspending the forcing beyond a threshold time, as done in Fig. 22a, relaminarization would have been attained. The controlled amplitude F-type forcing method is thus effective in inducing transition in the flow even at very low Reynolds numbers.

\section{Conclusions}

A new method is presented to build a continuous synthetic forcing capable of induce stochastic velocity perturbations with a prescribed energy level in wall-bounded parallel flows through receptivity mechanisms. The method is designed to provide a numerical framework for reproducing uncontrolled laminar-turbulent transition in wall-bounded parallel flows, characterised by high levels of external noise. Exploiting some concepts provided by linear stability and sensitivity analysis, such as the resolvent norm, we demonstrate that this method, which we refer to as F-type forcing method, is able to produce a stochastic velocity perturbation of prescribed energy level leading to turbulence. This goal is achieved by using a low-amplitude volume forcing composed of optimal forcing functions with different suitably chosen frequencies.

The method has been first tested in a linear framework using direct numerical simulations of the linearised Navier-Stokes equations, allowing a tuning of the numerical parameters. Then, direct numerical simulations of the fully nonlinear equations in the presence of the F-type volume forcing have been performed to test the robustness of the method. Subcritical transition to turbulence induced by the prescribed forcing has been investigated and compared to other transition scenarios, where deterministic (and unrealistic) perturbations are imposed for obtaining a fully turbulent flow.

Finally, the fully developed turbulent flow induced by the F-type method has been investigated. Low-order statistics and energy balance equations are practically unaffected by the continuous synthetic forcing. However, in the energy spectra a slight discrepancy with respect to literature data is retrieved, that can be easily avoided by suspending the forcing once a fully turbulent regime is attained. Thus, the F-type forcing method is modified by adding a controlled amplitude parameter based on runtime measured quantities, able to automatically damp the continuous forcing once the turbulent state is attained. Finally, the robustness of the controlled forcing amplitude F-type method has been demonstrated, by verifying its effectiveness in sustaining a transitional state even at very low Reynolds number.

The F-type method introduced here has proven to be a useful tool for studying uncontrolled transition in wall-bounded, parallel flows, such as the channel flow considered here. The same technique can be used without any modification to study non-deterministic transition to turbulence in a number of other closed flow configuration, avoiding the use of unstable modes for constructing the initial condition [31,53], as for instance done for the duct flow in Ref. [54]. The same volume forcing method could be also used in a spatially developing flow such as the boundary layer flow, by forcing a small part of the domain and letting the disturbance develop downstream. The F-type forcing method allows not only to study uncontrolled transition in a wide range of closed, wall bounded shear flows, but also provides a numerical framework for better understanding the onset and propagation of turbulence at its edge, the dynamics of incipients turbulent fronts [55], puffs and slugs [56] in pipe flow, as well as the establishment of direct percolation in the Couette flow [57].

\section{Acknowledgements}

This work was granted access to the HPC resources of IDRIS, TGCC and CINESunder the allocation A0052A06362 made by GENCI (Grand Equipement National deCalcul Intensif). The authors acknowledge the support of the French Agence Nationalede la Recherche (ANR) under grant DETAIL (ANR-15-CE29-0008-01). 


\section{Appendix A. Derivation of mean and turbulent kinetic energy balance equations}

We detail the derivation of Mean and Turbulent Kinetic Energy (MKE and TKE) balance equations in the framework of a temporal channel flow with F-type volume forcing. In the following we make use of the convention of summation over identical indices.

\section{A.1. MKE derivation}

Applying the time-averaging defined in equation (36) to equation (1) where the decomposition of the velocity field given in equations (34) and (35) has been used, one obtains:

$$
\frac{\partial \widehat{U_{i}}}{\partial t}=-\frac{\partial \widehat{U_{i} U_{j}}}{\partial x_{j}}-\frac{\partial \widehat{u_{i} u_{j}}}{\partial x_{j}}-\frac{\partial \widehat{P}}{\partial x_{i}}+\frac{1}{R e} \frac{\partial^{2} \widehat{U_{i}}}{\partial x_{j}^{2}}
$$

In order to recover an equation for the mean kinetic energy we multiply equation (A.1) by $U_{i}$, giving:

$$
\underbrace{\widehat{U}_{i} \frac{\partial \widehat{U}_{i}}{\partial t}}_{\mathrm{a}}=-\underbrace{\widehat{U}_{i} \frac{\partial \widehat{U}_{i} U_{j}}{\partial x_{j}}}_{\mathrm{b}}-\underbrace{\widehat{U}_{i} \frac{\partial \widehat{u_{i} u_{j}}}{\partial x_{j}}}_{\mathrm{c}}-\underbrace{\widehat{U_{i} \frac{\partial \widehat{P}}{\partial x_{i}}}}_{\mathrm{d}}+\underbrace{\widehat{U}_{i} \frac{1}{R e} \frac{\partial^{2} \widehat{U}_{i}}{\partial x_{j}^{2}}}_{\mathrm{e}}
$$

Developing all the terms one by one, we obtain:

$$
\begin{aligned}
& \text { (a) }=\frac{\partial}{\partial t}\left(\frac{\widehat{U_{i}^{2}}}{2}\right) \\
& \text { (b) }=\frac{\partial}{\partial x_{j}}\left(\frac{\widehat{U_{i}^{2}} \widehat{U_{j}}}{2}\right) \\
& \text { (c) }=\underbrace{\widehat{u_{i} u_{j}} \frac{\partial \widehat{U}_{i}}{\partial x_{j}}}_{\text {c.1 }}-\underbrace{\frac{\partial}{\partial x_{j}}\left(\widehat{u_{i} u_{j}} \widehat{U}_{j}\right)}_{\text {c.2 }} \\
& (d)=-\widehat{U}_{i} \frac{\partial \widehat{P}}{\partial x_{i}} \\
& \text { (e) }=\underbrace{\frac{1}{\operatorname{Re}} \frac{\partial}{\partial x_{j}}\left(\widehat{U}_{i} \frac{\partial \widehat{U}_{i}}{\partial x_{j}}\right)}_{\text {e. } 1}-\underbrace{\frac{1}{\operatorname{Re}\left(\frac{\partial \widehat{U}_{i}}{\partial x_{j}}\right)^{2}}}_{\text {e. } 2}
\end{aligned}
$$

which, rearranged into a single equation, provide the MKE balance equation for a generic incompressible Newtonian flow. In the framework of a temporal channel flow, the MKE equation can be reduced as follows:

$$
\underbrace{\frac{1}{2} \frac{\partial \widehat{U^{2}}}{\partial t}}_{\mathrm{a}}+\underbrace{\widehat{U} \Pi}_{\mathrm{d}}=+\underbrace{\widehat{u v} \frac{\partial \widehat{U}}{\partial y}}_{\mathrm{c} .1}-\underbrace{\frac{\partial \widehat{u v} \widehat{U}}{\partial y}}_{\mathrm{c} .2}+\underbrace{\frac{\partial}{\partial y}\left(\widehat{U} \frac{\partial \widehat{U}}{\partial y}\right)}_{\mathrm{e} .1}-\underbrace{\left(\frac{\partial \widehat{U}}{\partial y}\right)^{2}}_{\mathrm{e} .2},
$$

where the term (d) represents the power driving the flow, being $\Pi$ the streamwise pressure gradient. Time averaging and using some physical arguments [47], one can obtain the global transport equation of the MKE:

$$
U_{b} \tau_{w}=-\underbrace{\left[\widehat{u v} \frac{\partial \widehat{U}}{\partial y}\right]_{g}}_{P_{u v}}+\underbrace{\left[\left(\frac{\partial \widehat{U}}{\partial y}\right)^{2}\right]}_{D_{u}}
$$

The first term on the left represents the global input power, given by the pressure gradient force times the bulk velocity. The first term on the right, $P_{u v}$ (see (c.1)), is a source term for the MKE, allowing the energy to be transferred from the mean flow to the turbulent fluctuating field, thus providing the link between the MKE and the TKE. The last term on the right, $D_{u}$ (see (e.2)), represents the viscous dissipation due to the gradient of the mean flow. 


\section{A.2. TKE derivation}

We now derive the TKE balance equations under the same assumptions made in the previous section. Starting from equations (1), using the decomposition in equations (34) and (35), and then removing the equation for the mean state, we obtain the following equation for the perturbation evolution:

$$
\frac{\partial u_{i}}{\partial t}=-\widehat{U_{j}} \frac{\partial u_{i}}{\partial x_{j}}-u_{j} \frac{\partial \widehat{U}_{i}}{\partial x_{j}}-\frac{\partial p}{\partial x_{j}}+\frac{1}{R e} \frac{\partial^{2} u_{i}}{\partial x_{j}}-u_{j} \frac{\partial u_{i}}{\partial x_{j}}+u_{i} f_{i}
$$

Similarly to Reynolds-Orr equation in the linear-stability framework [31], we derive an evolution equation for the TKE by scalar multiplication of $u_{i}$ with the previous equation. By further rearranging equation (A.10), the following turbulent kinetic energy equation is obtained:

$$
\begin{aligned}
u_{i} \frac{\partial u_{i}}{\partial t} & =\underbrace{-u_{i} u_{j} \frac{\partial \widehat{U}_{i}}{\partial x_{j}}}_{P_{i i}} \underbrace{-\frac{1}{R e}\left(\frac{\partial u_{i}}{\partial x_{j}}\right)^{2}}_{\epsilon_{i i}} \\
& +\frac{\partial}{\partial x_{j}}[\underbrace{-\frac{1}{2} u_{i} u_{j} \widehat{U}_{j}}_{A_{i i}} \underbrace{-\frac{1}{2} u_{i} u_{i} u_{j}}_{Q_{i i}} \underbrace{-u_{i} p \delta_{i, j}}_{\phi_{i i}} \underbrace{+\frac{1}{R e} u_{i} \frac{\partial u_{i}}{\partial x_{j}}}_{D_{i i}}] \\
& +\underbrace{u_{i} f_{i}}_{F_{p}} .
\end{aligned}
$$

These terms are usually referred to as mean advection $\left(A_{i i}\right)$, production $\left(P_{i i}\right)$, transport by fluctuations $Q_{i i}$, pressure redistribution $\phi_{i i}$, viscous diffusion $D_{t}$, and turbulent dissipation $\epsilon_{i i}$ terms. The presence of the F-type forcing appears in the last term of (A.11), which is called $F_{p}$.

As for the MKE, we now rewrite the TKE in a temporal channel flow framework, taking the average in the spatially homogeneous directions:

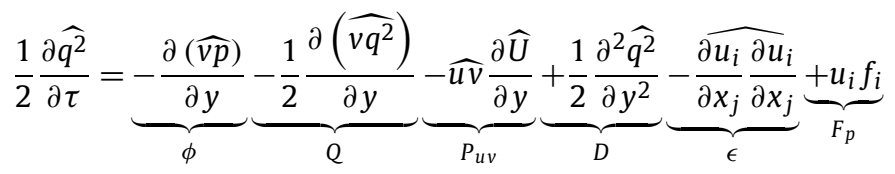

where $q^{2}=u_{i} u_{i}$. In the same way as for the MKE equations, time averaging and integration along $y$ of equations (A.14) allows for further simplifications [47], finally reading as:

$$
-P_{u v}+F_{p}=\epsilon
$$

\section{Appendix B. Supplementary material}

Supplementary material related to this article can be found online at https://doi.org/10.1016/j.jcp.2019.04.011.

\section{References}

[1] X. Wu, Inflow turbulence generation methods, Annu. Rev. Fluid Mech. 49 (1) (2017) 23-49, https://doi.org/10.1146/annurev-fluid-010816-060322.

[2] T.S. Lund, X. Wu, K.D. Squires, Generation of turbulent inflow data for spatially-developing boundary layer simulations, J. Comput. Phys. 140 (2) (1998) 233-258, https://doi.org/10.1006/jcph.1998.5882.

[3] N. Jarrin, S. Benhamadouche, D. Laurence, R. Prosser, A synthetic-eddy-method for generating inflow conditions for large-eddy simulations, Int. J. Heat Fluid Flow 27 (4) (2006) 585-593, https://doi.org/10.1016/j.ijheatfluidflow.2006.02.006, special Issue of The Fourth International Symposium on Turbulence and Shear Flow Phenomena - 2005.

[4] R.H. Kraichnan, Diffusion by a random velocity field, Phys. Fluids 13 (1) (1970) 22-31, https://doi.org/10.1063/1.1692799.

[5] S.B. Pope, Turbulent Flows, Cambridge University Press, 2000.

[6] Y.S. Kachanov, Physical mechanisms of laminar-boundary-layer transition, Annu. Rev. Fluid Mech. 26 (1) (1994) 411-482, https://doi.org/10.1146/ annurev.fl.26.010194.002211.

[7] H. Schlichting, K. Gersten, Boundary-Layer Theory, Springer, Berlin, Heidelberg, 2017.

[8] T. Sayadi, C.W. Hamman, P. Moin, Direct numerical simulation of complete h-type and k-type transitions with implications for the dynamics of turbulent boundary layers, J. Fluid Mech. 724 (2013) 480-509, https://doi.org/10.1017/jfm.2013.142.

[9] S. Berlin, A. Lundbladh, D. Henningson, Spatial simulations of oblique transition in a boundary layer, Phys. Fluids 6 (6) (1994) 1949-1951, https:// doi.org/10.1063/1.868200.

[10] J. Hoepffner, L. Brandt, D.S. Henningson, Transient growth on boundary layer streaks, J. Fluid Mech. 537 (2005) 91-100, https://doi.org/10.1017/ S0022112005005203. 
[11] P.S. Klebanoff, K.D. Tidstrom, L.M. Sargent, The three-dimensional nature of boundary-layer instability, J. Fluid Mech. 12 (1) (1962) 1-34, https:// doi.org/10.1017/S0022112062000014.

[12] J. Kendall, Experiments on boundary-layer receptivity to freestream turbulence, in: 36th AIAA Aerospace Sciences Meeting and Exhibit, American Institute of Aeronautics and Astronautics, 1998.

[13] M. Matsubara, P.H. Alfredsson, Disturbance growth in boundary layers subjected to free-stream turbulence, J. Fluid Mech. 430 (2001) 149-168, https:// doi.org/10.1017/s0022112000002810.

[14] L. Brandt, P. Schlatter, D.S. Henningson, Transition in boundary layers subject to free-stream turbulence, J. Fluid Mech. 517 (2004) 167-198, https:// doi.org/10.1017/S0022112004000941.

[15] R.G. Jacobs, P.A. Durbin, Simulations of bypass transition, J. Fluid Mech. 428 (2001) 185-212, https://doi.org/10.1017/S0022112000002469.

[16] J.W. Elder, An experimental investigation of turbulent spots and breakdown to turbulence, J. Fluid Mech. 9 (2) (1960) 235-246, https://doi.org/10.1017/ S0022112060001079.

[17] X. Wu, P. Moin, J.-P. Hickey, Boundary layer bypass transition, Phys. Fluids 26 (9) (2014) 091104, https://doi.org/10.1063/1.4893454.

[18] J. Lee, T. Zaki, Video: a computational laboratory for the study of transitional and turbulent boundary layers, in: 68th Annual Meeting of the APS Division of Fluid Dynamics - Gallery of Fluid Motion, American Physical Society, 2015.

[19] P. Klebanoff, Characteristics of turbulence in boundary layer with zero pressure gradient.

[20] S. Berlin, M. Wiegel, D.S. Henningson, Numerical and experimental investigations of oblique boundary layer transition, J. Fluid Mech. 393 (1999) 23-57, https://doi.org/10.1017/S002211209900511X.

[21] P. Schlatter, R. Örlü, Q. Li, G. Brethouwer, J.H.M. Fransson, A.V. Johansson, P.H. Alfredsson, D.S. Henningson, Turbulent boundary layers up to Re ${ }_{\theta}=2500$ studied through simulation and experiment, Phys. Fluids 21 (5) (2009) 051702, https://doi.org/10.1063/1.3139294

[22] M. Morkovin, Transition in open flow systems-a reassessment, Bull. Am. Phys. Soc. 39 (1994) 1882.

[23] T.A. Zang, S.E. Krist, Numerical experiments on stability and transition in plane channel flow, Theor. Comput. Fluid Dyn. 1 (1) (1989) 41-64, https:// doi.org/10.1007/BF00271421.

[24] N.D. Sandham, L. Kleiser, The late stages of transition to turbulence in channel flow, J. Fluid Mech. 245 (1992) 319-348, https://doi.org/10.1017/ S002211209200048X.

[25] S.J. Lee, T.A. Zaki, Simulations of natural transition in viscoelastic channel flow, J. Fluid Mech. 820 (2017) 232-262, https://doi.org/10.1017/jfm.2017.198.

[26] L. Kleiser, T.A. Zang, Numerical simulation of transition in wall-bounded shear flows, Annu. Rev. Fluid Mech. 23 (1) (1991) 495-537, https://doi.org/10. 1146/annurev.fl.23.010191.002431.

[27] S.C. Reddy, P.J. Schmid, J.S. Baggett, D.S. Henningson, On stability of streamwise streaks and transition thresholds in plane channel flows, J. Fluid Mech. 365 (1998) 269-303, https://doi.org/10.1017/S0022112098001323.

[28] N.J. Mullenix, D.V. Gaitonde, M.R. Visbal, Spatially developing supersonic turbulent boundary layer with a body-force-based method, AIAA J. 51 (8) (2013) 1805-1819, https://doi.org/10.2514/1.j051861.

[29] M. Klein, N. Chakraborty, S. Ketterl, A comparison of strategies for direct numerical simulation of turbulence chemistry interaction in generic planar turbulent premixed flames, Flow Turbul. Combust. 99 (3-4) (2017) 955-971, https://doi.org/10.1007/s10494-017-9843-9.

[30] S. Schmidt, M. Breuer, Source term based synthetic turbulence inflow generator for eddy-resolving predictions of an airfoil flow including a laminar separation bubble, Comput. Fluids 146 (2017) 1-22, https://doi.org/10.1016/j.compfluid.2016.12.023.

[31] P.J. Schmid, D.S. Henningson, Stability and Transition in Shear Flows, Springer, New York, 2001.

[32] P.J. Schmid, Nonmodal stability theory, Annu. Rev. Fluid Mech. 39 (1) (2007) 129-162, https://doi.org/10.1146/annurev.fluid.38.050304.092139.

[33] R. Moarref, M. Jovanovic, J. Tropp, B. McKeon, N. Sharma, A low-order decomposition of turbulent channel flow via resolvent analysis and convex optimization, Phys. Fluids 26 (51701) (2014) 1-7, https://eprints.soton.ac.uk/364749/.

[34] A. Sharma, R. Moarref, B. Mckeon, Scaling and interaction of self-similar modes in models of high-Reynolds number wall turbulence, Philos. Trans. R. Soc. A 375 (2089) (2017) 1-14, https://eprints.soton.ac.uk/402214/.

[35] J. Jiménez, P. Moin, The minimal flow unit in near-wall turbulence, J. Fluid Mech. 225 (1991) 213-240, https://doi.org/10.1017/S0022112091002033.

[36] J. Kim, P. Moin, R. Moser, Turbulence statistics in fully developed channel flow at low Reynolds number, J. Fluid Mech. 177 (1987) 133-166, https:// doi.org/10.1017/S0022112087000892.

[37] P. Schlatter, S. Stolz, L. Kleiser, LES of transitional flows using the approximate deconvolution model, Int. J. Heat Fluid Flow 25 (3) (2004) 549-558, https://doi.org/10.1016/j.ijheatfluidflow.2004.02.020.

[38] M. Sano, K. Tamai, A universal transition to turbulence in channel flow, Nat. Phys. 12 (3) (2016) 249-253, https://doi.org/10.1038/nphys3659.

[39] S.A. Orszag, Accurate solution of the Orr-Sommerfeld stability equation, J. Fluid Mech. 50 (04) (1971) 689, https://doi.org/10.1017/s0022112071002842.

[40] A. Jerri, The Shannon sampling theorem-its various extensions and applications: a tutorial review, Proc. IEEE 65 (11) (1977) 1565-1596, https:// doi.org/10.1109/proc.1977.10771.

[41] J.W.L. Paul, F. Fischer, S.G. Kerkemeier, nek5000 web page, http://nek5000.mcs.anl.gov, 2008.

[42] A. de Cheveigné, H. Kawahara, YIN, a fundamental frequency estimator for speech and music, J. Acoust. Soc. Am. (2002).

[43] M. Nishioka, S.I. A, Y. Ichikawa, An experimental investigation of the stability of plane Poiseuille flow, J. Fluid Mech. 72 (04) (1975) 731, https:// doi.org/10.1017/s0022112075003254.

[44] Y. Zhao, Z. Xia, Y. Shi, Z. Xiao, S. Chen, Constrained large-eddy simulation of laminar-turbulent transition in channel flow, Phys. Fluids 26 (9) (2014) 095103, https://doi.org/10.1063/1.4895589.

[45] Y. Zhao, Y. Yang, S. Chen, Evolution of material surfaces in the temporal transition in channel flow, J. Fluid Mech. 793 (2016) 840-876, https:// doi.org/10.1017/jfm.2016.152.

[46] P. Luchini, Reynolds-number-independent instability of the boundary layer over a flat surface: optimal perturbations, J. Fluid Mech. 404 (2000) 289-309, https://doi.org/10.1017/S0022112099007259.

[47] P. Ricco, C. Ottonelli, Y. Hasegawa, M. Quadrio, Changes in turbulent dissipation in a channel flow with oscillating walls, J. Fluid Mech. 700 (2012) 77-104, https://doi.org/10.1017/jfm.2012.97.

[48] R.D. Moser, J. Kim, N.N. Mansour, Direct numerical simulation of turbulent channel flow up to retau=590, Phys. Fluids 11 (4) (1999) 943-945, https:// doi.org/10.1063/1.869966.

[49] C.C. Holt, Forecasting seasonals and trends by exponentially weighted moving averages, Int. J. Forecast. 20 (1) (2004) 5-10, https://doi.org/10.1016/j. ijforecast.2003.09.015.

[50] T. Tsukahara, Y. Seki, H. Kawamura, D. Tochio, DNS of turbulent channel flow at very low Reynolds numbers, arXiv:1406.0248, 2014.

[51] P. Manneville, On the transition to turbulence of wall-bounded flows in general, and plane Couette flow in particular, Eur. J. Mech. B, Fluids 49 (2015) 345-362, https://doi.org/10.1016/j.euromechflu.2014.03.017.

[52] O. Iida, Y. Nagano, Flow Turbul. Combust. 60 (2) (1998) 193-213, https://doi.org/10.1023/a:1009999606355.

[53] T. Tatsumi, T. Yoshimura, Stability of the laminar flow in a rectangular duct, J. Fluid Mech. 212 (1) (1990) 437, https://doi.org/10.1017/ S002211209000204x.

[54] D. Biau, A. Bottaro, An optimal path to transition in a duct, Philos. Trans. R. Soc. A, Math. Phys. Eng. Sci. 367 (1888) (2009) 529-544, https://doi.org/ 10.1098/rsta.2008.0191. 
[55] B. Song, D. Barkley, B. Hof, M. Avila, Speed and structure of turbulent fronts in pipe flow, J. Fluid Mech. 813 (2017) 1045-1059, https://doi.org/10.1017/ jfm.2017.14.

[56] D. Barkley, Theoretical perspective on the route to turbulence in a pipe, J. Fluid Mech. 803 (2016), https://doi.org/10.1017/jfm.2016.465.

[57] G. Lemoult, L. Shi, K. Avila, S.V. Jalikop, M. Avila, B. Hof, Directed percolation phase transition to sustained turbulence in Couette flow, Nat. Phys. 12 (3) (2016) 254-258, https://doi.org/10.1038/nphys3675. 